ORNL-CTP-96-03

\title{
On the Mechanism of Open-Flavor Strong Decays ${ }^{\dagger}$
}

\author{
E.S.Ackleh, ${ }^{*}$ T.Barnes \\ Computational and Theoretical Physics Group \\ Oak Ridge National Laboratory, Oak Ridge, TN 37831-6373, and \\ Department of Physics and Astronomy, University of Tennessee, Knoxville, TN 37996-1200 \\ E.S.Swanson \\ Department of Physics, North Carolina State University, Raleigh, NC 27695-8202
}

\begin{abstract}
Open-flavor strong decays are mediated by $q \bar{q}$ pair production, which is known to occur dominantly with ${ }^{3} \mathrm{P}_{0}$ quantum numbers. The relation of the phenomenological ${ }^{3} \mathrm{P}_{0}$ model of these decays to "microscopic" QCD decay mechanisms has never been clearly established. In this paper we investigate $q \bar{q}$ meson decay amplitudes assuming pair production from the scalar confining interaction (sKs) and from one gluon exchange (OGE). sKs pair production predicts decay amplitudes of approximately the correct magnitude and $\mathrm{D} / \mathrm{S}$ amplitude ratios in $b_{1} \rightarrow \omega \pi$ and $a_{1} \rightarrow \rho \pi$ which are close to experiment. The OGE decay amplitude is found to be subdominant in most cases, a notable exception being ${ }^{3} \mathrm{P}_{0} \rightarrow{ }^{1} \mathrm{~S}_{0}+{ }^{1} \mathrm{~S}_{0}$. The full sKs + OGE amplitudes differ significantly from ${ }^{3} \mathrm{P}_{0}$ model predictions in some channels and can be distinguished experimentally, for example through an accurate comparison of the $\mathrm{D} / \mathrm{S}$ amplitude ratios in $b_{1} \rightarrow \omega \pi$ and $a_{1} \rightarrow \rho \pi$.
\end{abstract}

\footnotetext{
${ }^{\dagger}$ Dedicated to Atsje Barnes, 1 Aug 1993 - 13 Jan 1996.

${ }^{*}$ Current address: Hewlett-Packard Corporation, SIT-UNIX Team, M.S.906-SE3, 20 Perimeter Summit Blvd., Atlanta, GA 30319-1417.
} 


\section{INTRODUCTION: THE ${ }^{3} \mathbf{P}_{0}$ MODEL.}

\section{A. Summary of the ${ }^{3} \mathbf{P}_{0}$ model.}

Strong decays constitute a rather poorly understood area of hadron physics. A phenomenological model of hadron decays was developed in the 1970s by LeYaouanc et al [1 [3], which assumes as earlier suggested by Micu 4 that during a hadron decay a $q \bar{q}$ pair is produced from the vacuum with vacuum quantum numbers, $J^{P C}=0^{++}$. Since this corresponds to a ${ }^{3} \mathrm{P}_{0} q \bar{q}$ state, this is now generally referred to as the ${ }^{3} \mathrm{P}_{0}$ decay model. The ${ }^{3} \mathrm{P}_{0}$ pair production Hamiltonian for the decay of a $q \bar{q}$ meson $\mathrm{A}$ to mesons $\mathrm{B}+\mathrm{C}$ is usually written in a rather complicated form with explicit wavefunctions [5], which in the conventions of Geiger and Swanson [6] (to within an irrelevant overall phase) is

$$
\begin{gathered}
\left\langle B C\left|H_{I}\right| A\right\rangle=\gamma \iint \frac{d^{3} r d^{3} y}{(2 \pi)^{3 / 2}} e^{\frac{i}{2} \vec{P}_{B} \cdot \vec{r}} \Psi_{A}(\vec{r})\langle\vec{\sigma}\rangle_{q \bar{q}} \cdot\left(i \vec{\nabla}_{B}+i \vec{\nabla}_{C}+\vec{P}_{B}\right) \Psi_{B}^{*}\left(\frac{\vec{r}}{2}+\vec{y}\right) \Psi_{C}^{*}\left(\frac{\vec{r}}{2}-\vec{y}\right) \\
\delta\left(\vec{P}_{A}-\vec{P}_{B}-\vec{P}_{C}\right)
\end{gathered}
$$

for all quark and antiquark masses equal. The strength $\gamma$ of the decay interaction is regarded as a free constant and is fitted to data [9].

Studies of hadron decays using this model have been concerned almost exclusively with numerical predictions, and have not led to any fundamental modifications. Recent studies have considered changes in the spatial dependence of the pair production amplitude as a function of quark coordinates [5-8] but the fundamental decay mechanism is usually not addressed; this is widely believed to be a nonperturbative process, involving "flux tube breaking". There have been some studies of the decay mechanism which consider an alternative phenomenological model in which the $q \bar{q}$ pair is produced with ${ }^{3} \mathrm{~S}_{1}$ quantum numbers [10]; this possibility however is rejected by experiment [6]. In this paper we first review the phenomenological ${ }^{3} \mathrm{P}_{0}$ model and its predictions and develop a diagrammatic representation, following which we will test candidate QCD (quark-gluon) decay mechanisms for these strong decays. 
An equivalent formulation of the ${ }^{3} \mathrm{P}_{0}$ model regards the decays as due to an interaction Hamiltonian involving Dirac quark fields,

$$
H_{I}=g \int d^{3} x \bar{\psi} \psi
$$

which in the nonrelativistic limit gives matrix elements identical to (1) with the identification

$$
\gamma=\frac{g}{2 m_{q}}
$$

where $m_{q}$ is the quark mass of the produced pair. The operator $g \bar{\psi} \psi$ leads to the decay $(q \bar{q})_{A} \rightarrow(q \bar{q})_{B}+(q \bar{q})_{C}$ through the $b^{\dagger} d^{\dagger}$ term.

This model makes no reference to color, which if included would simply change the definition of the interaction strength $\gamma$; since $\gamma$ is fitted to data, this would not change the predictions for meson decays. An ad hoc feature of the ${ }^{3} \mathrm{P}_{0}$ model is to allow only diagrams in which the $q \bar{q}$ pair separate into different final hadrons. This was originally motivated by experiment (specifically the weakness of $\phi \rightarrow \rho \pi$ ); in the QCD-based current-current decay models that we shall discuss subsequently the absence of these hairpin diagrams is a natural consequence of the production of the $q \bar{q}$ pair in a color octet state.

To determine a decay rate we evaluate the matrix element of the decay Hamiltonian, which is of the form

$$
\left\langle B C\left|H_{I}\right| A\right\rangle=h_{f i} \delta(\vec{A}-\vec{B}-\vec{C})
$$

This $h_{f i}$ decay amplitude can be combined with relativistic phase space to give the differential decay rate, which is

$$
\frac{d \Gamma_{A \rightarrow B C}}{d \Omega}=2 \pi \frac{P E_{B} E_{C}}{M_{A}}\left|h_{f i}\right|^{2}
$$

where we have set $\vec{A}=\overrightarrow{0}$ and $P=|\vec{B}|=|\vec{C}|$. An equivalent result is quoted by Geiger and Swanson [6] as the "actual phase space" case of their equation (20). We shall usually quote results for the amplitude $\mathcal{M}_{L_{B C} S_{B C}}$, defined by

$$
\Gamma_{A \rightarrow B C}=2 \pi \frac{P E_{B} E_{C}}{M_{A}} \sum_{L S}\left|\mathcal{M}_{L S}\right|^{2} .
$$


For $S=0$ this $\mathcal{M}_{L S}$ is the coefficient of $Y_{L M}$ in $h_{f i}$, and in general is the amplitude $\left\langle J M\left(L_{B C}, S_{B C}\right) \mid B C\right\rangle$ in the $B C$ final state.

We have developed a diagrammatic technique for evaluating the $h_{f i}$ matrix element, which is discussed in detail for $\rho \rightarrow \pi \pi$ in the ${ }^{3} \mathrm{P}_{0}$ model in Appendix A. For $q \bar{q}$ meson decays there are two independent diagrams, shown below.
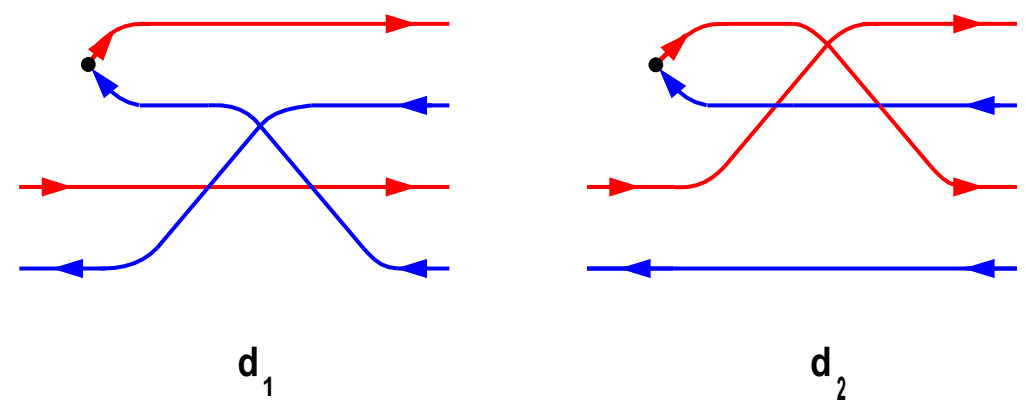

Figure 1. The two independent $q \bar{q}$ meson decay diagrams in the ${ }^{3} \mathrm{P}_{0}$ decay model.

\section{B. ${ }^{3} \mathbf{P}_{0}$ model decay rates for light $\mathbf{L}_{q \bar{q}}=0$ and $\mathbf{L}_{q \bar{q}}=1$ mesons.}

Since the ${ }^{3} \mathrm{P}_{0}$ model involves a free phenomenological interaction strength $\gamma$, comparison with experiment requires a fit to several rates (to determine both $\gamma$ and the wavefunction parameters) or a determination of amplitude ratios in decays with more than one partial wave, in which $\gamma$ cancels. Here we illustrate both applications; first we will evaluate the dominant two-body decay rates of light nonstrange mesons with $\mathrm{L}_{q \bar{q}}=0$ and $\mathrm{L}_{q \bar{q}}=1$, and subsequently we will evaluate $\mathrm{D} / \mathrm{S}$ ratios in the decays $b_{1} \rightarrow \omega \pi, a_{1} \rightarrow \rho \pi$ and $h_{1} \rightarrow \rho \pi$.

An $\mathcal{M}_{L S}$ decay amplitude in the ${ }^{3} \mathrm{P}_{0}$ model with $\mathrm{SHO}$ wavefunctions is proportional to a polynomial $\mathcal{P}_{L S}(x)$ in $x=P / \beta$ times an exponential,

$$
\mathcal{M}_{L S}=\frac{\gamma}{\pi^{1 / 4} \beta^{1 / 2}} \mathcal{P}_{L S}(x) e^{-x^{2} / 12} .
$$

For the cases considered here these polynomials are

$$
\mathcal{P}_{10}^{\left({ }^{3} \mathrm{~S}_{1} \rightarrow{ }^{1} \mathrm{~S}_{0}+{ }^{1} \mathrm{~S}_{0}\right)}=-\frac{2^{5}}{3^{3}} x
$$




$$
\begin{aligned}
& \mathcal{P}_{20}^{\left({ }^{3} \mathrm{P}_{2} \rightarrow{ }^{1} \mathrm{~S}_{0}+{ }^{1} \mathrm{~S}_{0}\right)}=+\frac{2^{6}}{3^{4} 5^{1 / 2}} x^{2} \\
& \mathcal{P}_{21}^{\left({ }^{3} \mathrm{P}_{2} \rightarrow{ }^{3} \mathrm{~S}_{1}+{ }^{1} \mathrm{~S}_{0}\right)}=-\frac{2^{11 / 2}}{3^{7 / 2} 5^{1 / 2}} x^{2} \\
& \mathcal{P}_{01}^{\left({ }^{3} \mathrm{P}_{1} \rightarrow{ }^{3} \mathrm{~S}_{1}+{ }^{1} \mathrm{~S}_{0}\right)}=+\frac{2^{5}}{3^{5 / 2}}\left(1-\frac{2}{9} x^{2}\right) \\
& \mathcal{P}_{21}^{\left({ }^{3} \mathrm{P}_{1} \rightarrow{ }^{3} \mathrm{~S}_{1}+{ }^{1} \mathrm{~S}_{0}\right)}=-\frac{2^{11 / 2}}{3^{9 / 2}} x^{2} \\
& \mathcal{P}_{00}^{\left({ }^{3} \mathrm{P}_{0} \rightarrow{ }^{1} \mathrm{~S}_{0}+{ }^{1} \mathrm{~S}_{0}\right)}=+\frac{2^{9 / 2}}{3^{2}}\left(1-\frac{2}{9} x^{2}\right) \\
& \mathcal{P}_{01}^{\left({ }^{1} \mathrm{P}_{1} \rightarrow{ }^{3} \mathrm{~S}_{1}+{ }^{1} \mathrm{~S}_{0}\right)}=-\frac{2^{9 / 2}}{3^{5 / 2}}\left(1-\frac{2}{9} x^{2}\right) \\
& \mathcal{P}_{21}^{\left({ }^{1} \mathrm{P}_{1} \rightarrow{ }^{3} \mathrm{~S}_{1}+{ }^{1} \mathrm{~S}_{0}\right)}=-\frac{2^{6}}{3^{9 / 2}} x^{2} .
\end{aligned}
$$

For physical decays there is an additional multiplicative flavor factor, as discussed in Appendix A. In figure 2 we show these decay rates for physical mesons for a wide range of wavefunction length scales $\beta$ (defined in App.A).

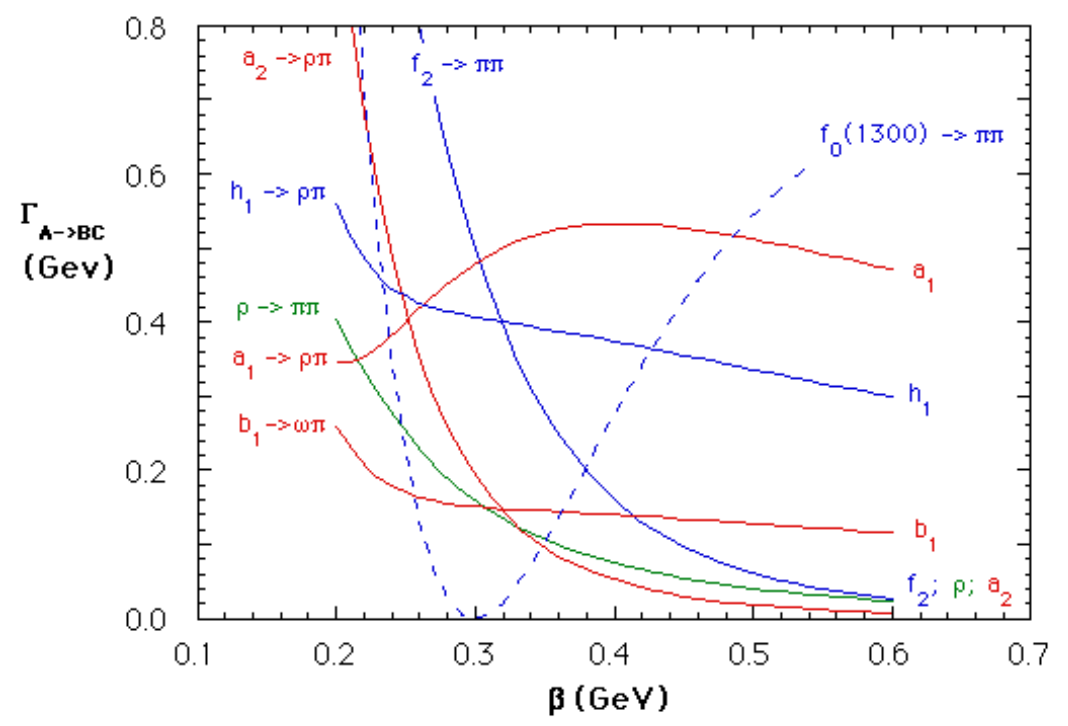

Figure 2. Representative light $q \bar{q}$ meson decay rates in the ${ }^{3} \mathrm{P}_{0}$ decay model. Parameters $\beta$ variable, $\gamma=0.5$. 
We set $\gamma=0.5$ in the figure because this is near the optimum fitted value. A value of $\beta \approx 0.35-0.4 \mathrm{GeV}$ evidently gives a reasonably accurate description of the relative decay rates (see also Table 1 below). Many recent quark model studies of meson [5] 8nd baryon 11] decays use a value of $\beta=0.4 \mathrm{GeV}$, at the upper end of this range.

A simple estimate of the optimum $\beta$ for these SHO wavefunctions follows if we require maximum overlap with Coulomb plus linear wavefunctions $\Psi$. With typical quark model parameters of $\alpha_{s}=0.6, b=0.18 \mathrm{GeV}^{2}$ and $m_{q}=0.33 \mathrm{GeV}$, the overlap $\left|\int \Psi^{*} \psi_{S H O} d^{3} x\right|^{2}$ is maximum at $\beta=0.316 \mathrm{GeV}$ for $\mathrm{L}_{q \bar{q}}=0$ (99.4\% overlap) and $\beta=0.274 \mathrm{GeV}$ for $\mathrm{L}_{q \bar{q}}=1$ (99.6\% overlap). Curiously, this $\beta$ would imply a narrow $f_{0}(1300)$ in the ${ }^{3} P_{0}$ decay model. Comparison with figure 2 shows that these $\beta$ estimates are similar to the values required to describe decays, although there is some evidence of a systematic discrepancy, $\beta_{\text {decay }} / \beta_{\text {Cou. }+ \text { lin. }} \approx 1.2$

\begin{tabular}{||c||c|c|c|c||}
\hline \multicolumn{5}{||c||}{ Table $1 .{ }^{3} \mathrm{P}_{0}$ Model Fit to Light Meson Decay Rates. } \\
\hline Decay & Expt. [12] & ${ }^{3} \mathrm{P}_{0}$ Theory & D/S Expt [12].13 & D/S ${ }^{3} \mathrm{P}_{0}$ Theory \\
\hline$\rho \rightarrow \pi \pi$ & $151 . \mathrm{MeV}$ & $79 . \mathrm{MeV}$ & & \\
\hline$f_{2} \rightarrow \pi \pi$ & $157 . \mathrm{MeV}$ & $170 . \mathrm{MeV}$ & & \\
\hline$a_{2} \rightarrow \rho \pi$ & $72 . \mathrm{MeV}$ & $54 . \mathrm{MeV}$ & & \\
\hline$a_{1} \rightarrow \rho \pi$ & $400 . \mathrm{MeV}$ & $545 . \mathrm{MeV}$ & $-0.09(2)$ & -0.154 \\
\hline$b_{1} \rightarrow \omega \pi$ & $142 . \mathrm{MeV}$ & $143 . \mathrm{MeV}$ & $+0.260(35)$ & +0.292 \\
\hline$h_{1} \rightarrow \rho \pi$ & $360 . \mathrm{MeV}$ & $383 . \mathrm{MeV}$ & - & -0.111 \\
\hline $\mathrm{K}_{0}^{*}(1430) \rightarrow \mathrm{K} \pi$ & $287 . \mathrm{MeV}$ & $166 . \mathrm{MeV}$ & & \\
\hline$f_{0} \rightarrow \pi \pi$ & $150-400 \mathrm{MeV}($ not fitted $)$ & $271 . \mathrm{MeV}$ & & \\
\hline
\end{tabular}

In Table 1 we show the result of a fit to a representative set of seven well established light S- and P-wave $q \bar{q}$ meson decay rates, with $\beta$ and $\gamma$ taken as free parameters. The masses assumed were $M_{\pi}=0.138 \mathrm{GeV}, M_{\mathrm{K}}=0.496 \mathrm{GeV}, M_{\rho}=0.77 \mathrm{GeV}, M_{\omega}=0.782 \mathrm{GeV}$, 
$M_{h_{1}}=1.17 \mathrm{GeV}, M_{a_{1}}=1.23 \mathrm{GeV}, M_{b_{1}}=1.231 \mathrm{GeV}, M_{f_{0}}=1.3 \mathrm{GeV}, M_{a_{2}}=1.318 \mathrm{GeV}$ and $M_{\mathrm{K}_{0}^{*}}=1.429 \mathrm{GeV}$. All but $\mathrm{K}_{0}^{*}(1430) \rightarrow \mathrm{K} \pi$ are nonstrange systems, which we chose to avoid parameter differences due to strange quarks. The $\mathrm{K}_{0}^{*}(1430)$ is included because we found that it is quite important to include a light ${ }^{3} \mathrm{P}_{0} q \bar{q}$ decay, since the rate ${ }^{3} \mathrm{P}_{0} \rightarrow{ }^{1} \mathrm{~S}_{0}+{ }^{1} \mathrm{~S}_{0}$ is quite sensitive to $\beta$, and the $\mathrm{K}_{0}^{*}(1430)$ is the only well established ${ }^{3} \mathrm{P}_{0}$ resonance. On fitting these widths using Eqs.(6-15) (minimizing $\left.\sum_{i=1}^{7}\left(\Gamma_{A \rightarrow B C}^{\text {thy. }} / \Gamma_{A \rightarrow B C}^{\text {expt. }}-1\right)^{2}\right)$, we find the parameters

$$
\begin{gathered}
\beta=0.397 \mathrm{GeV} \\
\gamma=0.506 .
\end{gathered}
$$

Clearly the most important discrepancy in the table is $\rho \rightarrow \pi \pi$, which is well known to be a problem relative to the decays of $\mathrm{P}$-wave $q \bar{q}$ mesons in this model. The $\mathrm{K}_{0}^{*}(1430)$ width is also rather smaller than experiment.

If we attempt constrained fits with fixed $\beta$, we find serious disagreement with experiment for even moderate changes away from $\beta=0.4 \mathrm{GeV}$. For example, at $\beta=0.3 \mathrm{GeV}$ we find an $f_{2}$ broader than the $a_{1}$ and $h_{1}$ and a $\mathrm{K}_{0}^{*}(1430)$ width near zero. Conversely, if we increase $\beta$ to $0.5 \mathrm{GeV}$ we find quite small partial widths for $\rho \rightarrow \pi \pi, a_{2} \rightarrow \rho \pi$ and $f_{2} \rightarrow \pi \pi$ relative to the $b_{1}, a_{1}$ and $h_{1}$, on average only 0.31 of $b_{1} \rightarrow \omega \pi$; experimentally the ratio is 0.89 .

The interesting question of the width of an $f_{0}(1300) q \bar{q}$ state is unfortunately problematical in the ${ }^{3} \mathrm{P}_{0}$ model, since like $\mathrm{K}_{0}^{*}(1430) \rightarrow \mathrm{K} \pi$ this rate has a node near $\beta=0.30 \mathrm{GeV}$, and increases rapidly with $\beta$ above the node. For our fitted parameters we find $\Gamma_{f_{0}(1300) \rightarrow \pi \pi}=$ $271 \mathrm{MeV}$, but comparison of the predicted and observed $\mathrm{K}_{0}^{*}(1430)$ widths suggests that the actual $f_{0}(1300)$ partial width to $\pi \pi$ is $\approx 450 \mathrm{MeV}$. We shall subsequently show that the transition ${ }^{3} \mathrm{P}_{0} \rightarrow{ }^{1} \mathrm{~S}_{0}+{ }^{1} \mathrm{~S}_{0}$ has an unusually large OGE decay amplitude, which may explain why the light scalars $f_{0}(1300)$ and $\mathrm{K}_{0}^{*}(1430)$ are broad states despite the node in the ${ }^{3} \mathrm{P}_{0}$ decay amplitude. 


\section{C. ${ }^{3} \mathbf{P}_{0}$ results for $\mathrm{D} / \mathrm{S}$ ratios}

Sensitive tests of decay models are possible in decays with more than one partial wave, because one can measure the relative phases as well as the magnitudes of the decay amplitudes in these processes.

Multiamplitude decays require at least one final meson to have nonzero spin. For the decays considered here the relevant final states are $\rho \pi$ and $\omega \pi$; other possibilities are excluded by phase space. These final states have $\mathrm{S}^{P}=1^{+}$, so of all the light $\mathrm{L}_{q \bar{q}}=0$ and $\mathrm{L}_{q \bar{q}}=1$ mesons only the $1^{+}$mesons have more than one partial wave; these decay to both $\mathrm{S}$ - and $\mathrm{D}$ wave vector + pseudoscalar final states. On increasing $\mathrm{L}_{q \bar{q}}$ we next encounter multiamplitude decays in the $\mathrm{D}$ states, for example in $\pi_{2} \rightarrow \rho \pi(\mathrm{P}, \mathrm{F})$ [6] and $\pi_{2} \rightarrow f_{2} \pi(\mathrm{S}, \mathrm{D}, \mathrm{G})$.

Here we will calculate the $\mathrm{D} / \mathrm{S}$ amplitude ratios in the decays $b_{1} \rightarrow \omega \pi$ and $a_{1} \rightarrow \rho \pi$. $\mathrm{D} / \mathrm{S}$ for $h_{1} \rightarrow \rho \pi$, which has not been measured, is theoretically equal to $b_{1} \rightarrow \omega \pi$ to within

small phase space differences, since these are both ${ }^{1} \mathrm{P}_{1} \rightarrow{ }^{3} \mathrm{~S}_{1}+{ }^{1} \mathrm{~S}_{0}$ decays. The $b_{1}$ is clearly the most attractive of these experimentally, due to the smaller widths of the $b_{1}$ and $\omega$.

We assume an initial polarization $b_{1}^{+}\left(\mathrm{J}_{z}=+1\right)$ and determine the $h_{f i}$ matrix element to a final state with a specific $\omega$ polarization that allows both $\mathrm{L}$ values; we choose $\omega\left(\mathrm{S}_{z}=+1\right)$. Using the diagrammatic techniques of Appendix A, we find that the two diagrams $d_{1}$ and $d_{2}$ give equal contributions, and the total $h_{f i}$ is

$$
\left.h_{f i}\right|_{b_{1}^{+}(+\hat{z}) \rightarrow \omega(+\hat{z}) \pi^{+}}=-\frac{\gamma}{\pi^{1 / 4} \beta^{1 / 2}} \frac{2^{4}}{3^{5 / 2}}\left\{\left(1-\frac{2}{9} x^{2}\right) Y_{00}(\Omega)+\frac{2}{3^{2} 5^{1 / 2}} x^{2} Y_{20}(\Omega)\right\} e^{-x^{2} / 12} .
$$

This matrix element determines the relative $S$ and D amplitudes in the $|\omega \pi\rangle$ final state; since the $\mathrm{J}^{P}=1^{+}|\omega \pi\rangle$ state is of the form

$$
\left.\left.|\omega \pi\rangle=\left.a_{S}\right|^{3} \mathrm{~S}_{1}\right\rangle+\left.a_{D}\right|^{3} \mathrm{D}_{1}\right\rangle
$$

it follows from a Clebsch-Gordon decomposition that the amplitude to find a $(+\hat{z})$-polarized $\omega$ in an $\omega \pi$ pair with decay direction $\Omega$ must be

$$
\left\langle\omega(+\hat{z})_{\Omega} \pi_{-\Omega} \mid \omega \pi\right\rangle=a_{S} Y_{00}(\Omega)+a_{D} \sqrt{\frac{1}{10}} Y_{20}(\Omega)
$$


We can therefore read the $a_{D} / a_{S}$ ratio directly from the $h_{f i}$ matrix element (18), which gives

$$
\left.\frac{a_{D}}{a_{S}}\right|_{b_{1} \rightarrow \omega \pi}=+\frac{2^{3 / 2}}{3^{2}} \frac{x^{2}}{\left(1-\frac{2}{9} x^{2}\right)} .
$$

This is equivalent to the result $(4.11,12)$ of LeYaouanc et al. [1] when one specializes their result to $R_{b_{1}}=R_{\omega}=R_{\pi}$, which is our $1 / \beta$. This $\mathrm{D} / \mathrm{S}$ ratio is also implicit in $(14,15)$. Since the strength of the pair production amplitude and some of the momentum dependence in the overlap integrals cancel out in this ratio, there is less systematic uncertainty than in the decay rates. Proceeding similarly for $a_{1} \rightarrow \rho \pi$ we find

$$
\left.\frac{a_{D}}{a_{S}}\right|_{a_{1} \rightarrow \rho \pi}=-\frac{2^{1 / 2}}{3^{2}} \frac{x^{2}}{\left(1-\frac{2}{9} x^{2}\right)} .
$$

The ratio of $\mathrm{D} / \mathrm{S}$ ratios in these decays is especially interesting in the ${ }^{3} \mathrm{P}_{0}$ model because all the dependence on the spatial wavefunctions cancels. We then find

$$
\frac{\left.\frac{a_{D}}{a_{S}}\right|_{a_{1} \rightarrow \rho \pi}}{\left.\frac{a_{D}}{a_{S}}\right|_{b_{1} \rightarrow \omega \pi}}=-\frac{1}{2} .
$$

Experimentally these ratios are

$$
\left.\frac{a_{D}}{a_{S}}\right|_{b_{1} \rightarrow \omega \pi}=+0.260 \pm 0.035
$$

and from a recent ARGUS measurement 13

$$
\left.\frac{a_{D}}{a_{S}}\right|_{a_{1} \rightarrow \rho \pi}=-0.09 \pm 0.02
$$

so the ratio is

$$
\frac{\left.\frac{a_{D}}{a_{S}}\right|_{a_{1} \rightarrow \rho \pi}}{\left.\frac{a_{D}}{a_{S}}\right|_{b_{1} \rightarrow \omega \pi}}=-0.35 \pm 0.09 .
$$

Theory and experiment for these D/S ratios are compared in figure 3; a best fit to these D/S ratios alone gives $\beta=0.448 \mathrm{GeV}$, for which $(\mathrm{D} / \mathrm{S})_{b_{1} \rightarrow \omega \pi}=+0.219$ and $(\mathrm{D} / \mathrm{S})_{a_{1} \rightarrow \rho \pi}=-0.115$. (The small theoretical departure from $-1 / 2$ is due to phase space differences.) 
The experimental ratio (26) is approximately consistent with the ${ }^{3} \mathrm{P}_{0}$ prediction but shows the need for a more accurate experimental determination, especially in $a_{1} \rightarrow \rho \pi$. This important $\mathrm{D} / \mathrm{S}$ ratio has only been measured in one experiment; an improved value would allow an interesting test of the ${ }^{3} \mathrm{P}_{0}$ model, since the OGE decay mechanism predicts a departure of the ratio (23) from $-1 / 2$; we will discuss this in the next section.

In summary, the decay rates of light $\mathrm{L}_{q \bar{q}}=0$ and $\mathrm{L}_{q \bar{q}}=1$ mesons (figure 2) support $\beta \approx 0.40 \mathrm{GeV}$, whereas the $\mathrm{D} / \mathrm{S}$ amplitude ratios in $b_{1} \rightarrow \omega \pi$ and $a_{1} \rightarrow \rho \pi$ (figure 3 ) suggest a larger value, $\beta \approx 0.45 \mathrm{GeV}$. These are both significantly larger than the $\beta \approx 0.3 \mathrm{GeV}$ which gives the maximum overlap with Coulomb plus linear wavefunctions.

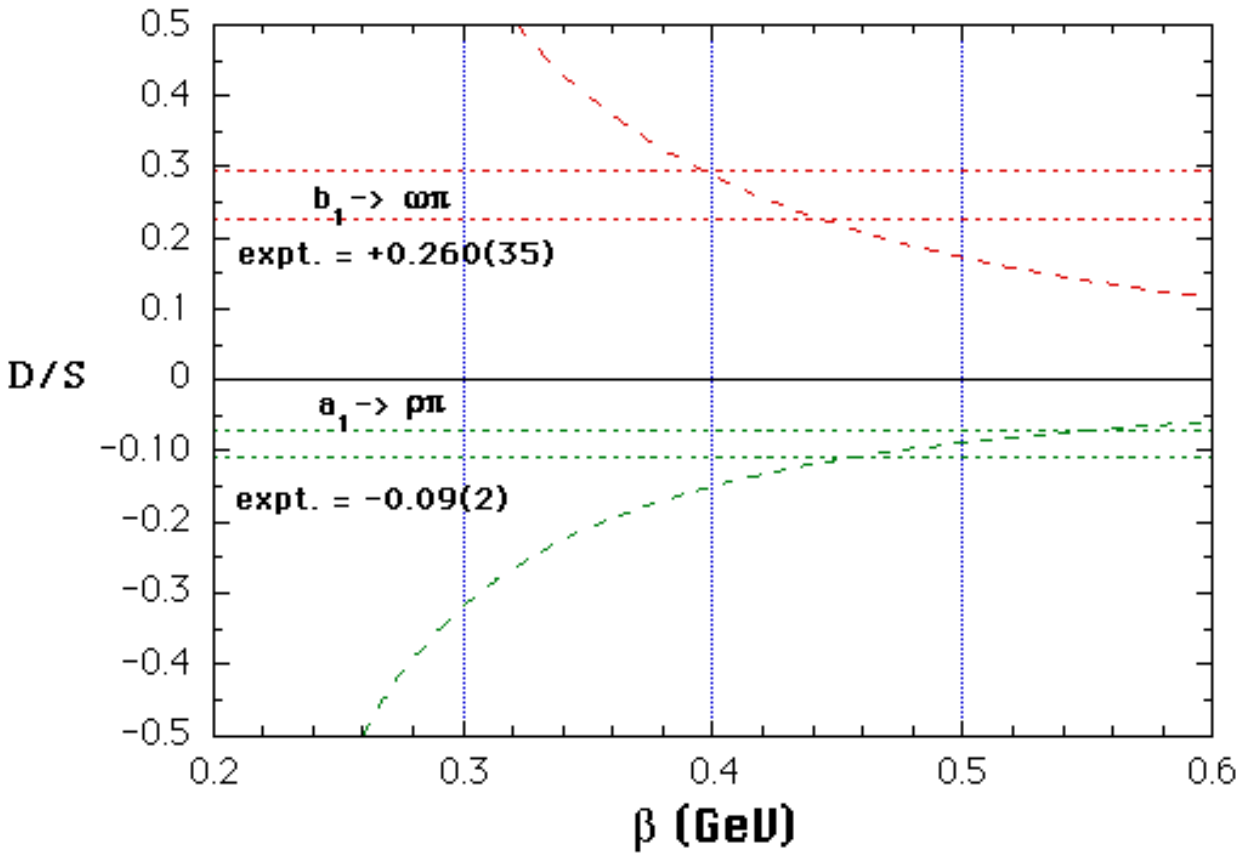

Figure 3. D/S amplitude ratios in $b_{1} \rightarrow \omega \pi$ and $a_{1} \rightarrow \rho \pi$ with ${ }^{3} \mathrm{P}_{0}$ model predictions.

\section{II. "MICROSCOPIC" DECAY MODELS}




\section{A. Candidate pair production Hamiltonians.}

In microscopic decay models one attempts to describe hadron strong decays in terms of quark and gluon degrees of freedom. The quark-gluon decay mechanism should give similar predictions to the reasonably accurate ${ }^{3} \mathrm{P}_{0}$ model, and should determine the strength $\gamma$ of the ${ }^{3} \mathrm{P}_{0}$ interaction in terms of fundamental QCD parameters. There has been little previous work in this area. One exception is the study of open charm decays of $c \bar{c}$ resonances by Eichten et al. [14], who assumed that decays are due to pair production from the confining interaction. We will confirm this assumption for light quarkonia in most but not all cases. Eichten et al. however assumed that confinement is a Lorentz vector interaction; this is now believed to be incorrect, and the currently accepted scalar form leads to quite different matrix elements.

We begin by assuming that these strong decays are driven by the same interquark Hamiltonian which determines the spectrum, and that it incorporates scalar confinement and one gluon exchange. These interactions and their associated decay amplitudes are undoubtedly all present and should be added coherently. We will determine decay rates for the two-body decays discussed in the previous section, assuming the appropriate field-theoretic generalization of the usual interquark Hamiltonian.

The current-current interactions due to the scalar confining interaction and OGE can be written in the generic form

$$
H_{I}=\frac{1}{2} \iint d^{3} x d^{3} y J^{a}(\vec{x}) \cdot K(|\vec{x}-\vec{y}|) \cdot J^{a}(\vec{y})
$$

The current $J^{a}$ in (27) is assumed to be a color octet. The currents $J$ (with the color dependence $\lambda^{a} / 2$ factored out) and the kernels $K(r)$ for the interactions are

$$
J= \begin{cases}\bar{\psi} \psi & \text { scalar confining interaction } \\ \psi^{\dagger} \psi & \text { color Coulomb OGE } \\ (\bar{\psi} \vec{\gamma} \psi)_{\mathrm{T}} & \text { transverse OGE }\end{cases}
$$




$$
K= \begin{cases}+\frac{3}{4}\left(b r+S_{0}\right) & \text { scalar confining interaction } \\ +\alpha_{s} / r & \text { color Coulomb OGE } \\ -\alpha_{s} / r & \text { transverse OGE }\end{cases}
$$

The scalar confinement kernel is normalized so that $b r+S_{0}$ is the static scalar potential of a color-singlet $q \bar{q}$ pair.

We refer to this general type of interaction as a JKJ decay model, and to the specific

cases considered here as $\mathrm{sKs}, \mathrm{j}^{0} \mathrm{Kj}^{0}$ and $\mathrm{j}^{\mathrm{T}} \mathrm{Kj}^{\mathrm{T}}$ interactions. The decay Hamiltonian assumed by Eichten et al. [14] in their Eq. (3.2) is a special case of our form (27) with a $\mathrm{j}^{0} \mathrm{Kj}^{0}$ interaction.

\section{B. JKJ A $\rightarrow$ BC matrix elements: general results.}

The $\mathrm{A} \rightarrow \mathrm{BC}$ decay matrix element of the JKJ Hamiltonian (27) involves a pair-production current matrix element $\langle q \bar{q}|J| 0\rangle$ times a scattering matrix element $\left\langle q_{f}|J| q_{i}\right\rangle$. Diagrammatically this corresponds to an interaction between an initial line and the produced pair, and there are four such diagrams (see figure 4). We label these using the ${ }^{3} \mathrm{P}_{0}$ quark line diagram labels $d_{1}$ and $d_{2}$, with an additional $q$ or $\bar{q}$ subscript denoting which initial line the produced pair interacts with.

We specialize to the diagram $d_{1 q}$ for illustration. As in the ${ }^{3} \mathrm{P}_{0}$ model (Appendix A) there is a Fermi "signature" phase, a flavor factor, and a spin+space overlap integral,

$$
\left\langle B C\left|H_{I}\right| A\right\rangle_{d_{1 q}}=I_{\text {signature }} \cdot I_{\text {color }} \cdot I_{\text {flavor }} \cdot \mathbf{I}_{\text {spin }+ \text { space }}
$$

where

$$
\mathbf{I}_{\text {spin }+ \text { space }}=I_{\text {spin }+ \text { space }} \delta(\vec{A}-\vec{B}-\vec{C}) .
$$



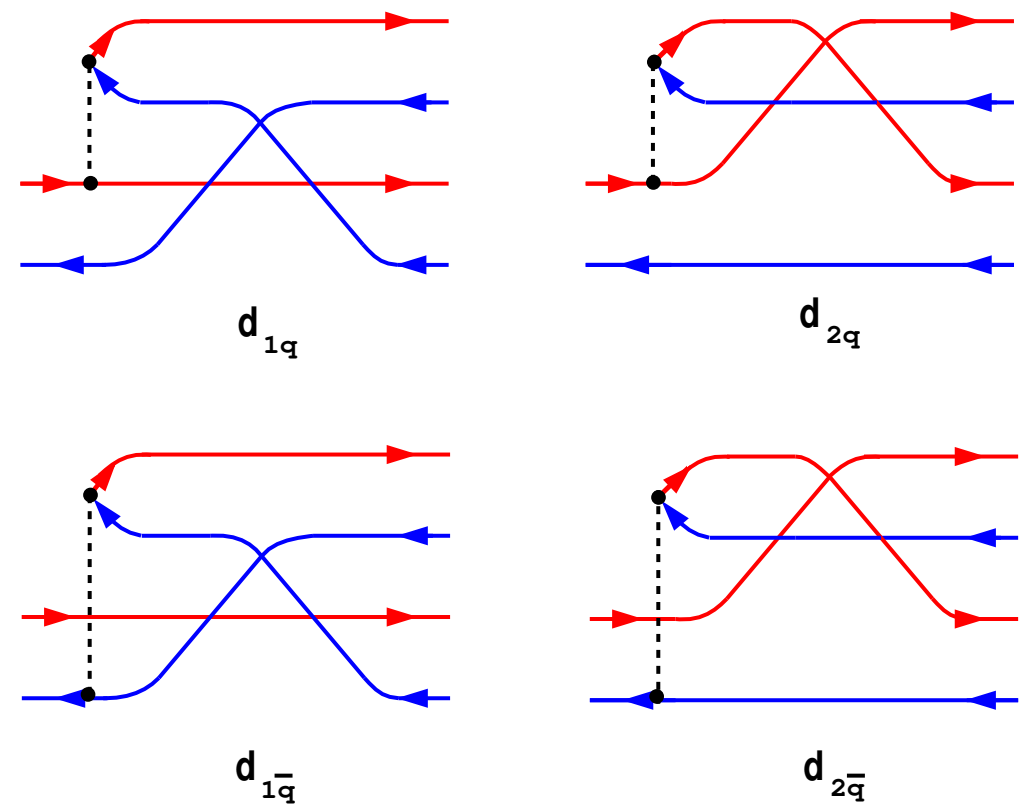

Figure 4. The four independent $q \bar{q}$ meson decay diagrams in JKJ decay models.

The signature is again $I_{\text {signature }}=(-1)^{3}$ because there are three line crossings. A new feature due to our explicit treatment of color is a color matrix element; the color factor for this diagram is

$$
I_{\text {color }}=\frac{1}{3^{3 / 2}} \operatorname{Tr}\left\{\frac{\lambda^{a}}{2} \frac{\lambda^{a}}{2}\right\}=+\frac{2^{2}}{3^{3 / 2}}
$$

and is the same for diagram $d_{2 q}$ and opposite for $d_{1 \bar{q}}$ and $d_{2 \bar{q}}$. The flavor and spin factors are specific to the reaction and will be discussed subsequently. The $d_{1 q}$ spatial overlap integral analogous to (A5) is

$$
\begin{gathered}
\mathbf{I}_{\text {space }}\left(d_{1 q}\right)=2 ! \iint d^{3} x d^{3} y \frac{1}{2} K(|\vec{x}-\vec{y}|) \iiint d^{3} a d^{3} b d^{3} c \phi_{A}(2 \vec{a}-\vec{A}) \phi_{B}^{*}(2 \vec{b}-\vec{B}) \phi_{C}^{*}(2 \vec{c}-\vec{C}) \\
\delta(\vec{A}-\vec{a}-\vec{B}+\vec{b})\langle c|J(\vec{x})| a\rangle\langle b \vec{c}|J(\vec{y})| 0\rangle,
\end{gathered}
$$

where the matrix elements of the currents (with $\Gamma=\gamma^{0}, \gamma^{i}, \mathrm{I}$ ) are

$$
\left\langle q^{\prime}|J(\vec{x})| q\right\rangle=+\frac{1}{(2 \pi)^{3}} \frac{m_{q}}{\sqrt{E_{q} E_{q^{\prime}}}} e^{i\left(q^{\prime}-q\right) \cdot x}\left[\bar{u}_{q^{\prime} s^{\prime}} \Gamma u_{q s}\right]
$$




$$
\left\langle\bar{q}^{\prime}|J(\vec{x})| \bar{q}\right\rangle=-\frac{1}{(2 \pi)^{3}} \frac{m_{q}}{\sqrt{E_{\bar{q}} E_{\bar{q}^{\prime}}}} e^{i\left(\bar{q}^{\prime}-\bar{q}\right) \cdot x}\left[\bar{v}_{\bar{q} \bar{s}} \Gamma v_{\bar{q}^{\prime} \bar{s}^{\prime}}\right]
$$

and

$$
\langle q \bar{q}|J(\vec{y})| 0\rangle=\frac{1}{(2 \pi)^{3}} \frac{m_{q}}{\sqrt{E_{q} E_{\bar{q}}}} e^{i(q+\bar{q}) \cdot y}\left[\bar{u}_{q s} \Gamma v_{\bar{q} \bar{s}}\right] .
$$

With these substitutions, and introducing a Fourier transformed kernel

$$
\mathcal{K}(Q)=\int d^{3} x e^{i \vec{Q} \cdot \vec{r}} K(r)
$$

we find

$$
\begin{aligned}
I_{\text {space }}\left(d_{1 q}\right)= & \frac{1}{(2 \pi)^{3}} \iint d^{3} a d^{3} c \phi_{A}(2 \vec{a}-\vec{A}) \phi_{B}^{*}(2 \vec{a}-2 \vec{C}-\vec{B}) \phi_{C}^{*}(2 \vec{c}-\vec{C}) \\
& \frac{m_{q}^{2}}{\sqrt{E_{a} E_{b} E_{c} E_{\bar{c}}}}\left[\bar{u}_{b s_{b}} \Gamma v_{\bar{c} s_{\bar{c}}}\right] \mathcal{K}(|\vec{a}-\vec{c}|)\left[\bar{u}_{c s_{c}} \Gamma u_{a s_{a}}\right]
\end{aligned}
$$

and there are implicit momentum constraints $\vec{b}=\vec{a}-\vec{C}$ and $\overrightarrow{\vec{c}}=\vec{C}-\vec{c}$ from the spatial integrations in (33) and the meson wavefunctions.

As in the ${ }^{3} \mathrm{P}_{0}$ model we take the nonrelativistic limit of this interaction for our decay amplitude, although it would be interesting in future work to investigate the effect of keeping the full relativistic amplitude (38). We also specialize to the rest frame, $\vec{A}=0$ and $\vec{B}=-\vec{C}$. These substitutions give our final general result for the $d_{1 q}$ overlap integral,

$$
\begin{aligned}
I_{\text {space }}\left(d_{1 q}\right)= & \frac{1}{(2 \pi)^{3}} \iint d^{3} a d^{3} c \phi_{A}(2 \vec{a}) \phi_{B}^{*}(2 \vec{a}+\vec{B}) \phi_{C}^{*}(2 \vec{c}+\vec{B}) \\
& {\left[\bar{u}_{b s_{b}} \Gamma v_{\bar{c}_{\bar{c}}}\right] \mathcal{K}(|\vec{a}-\vec{c}|)\left[\bar{u}_{c s_{c}} \Gamma u_{a s_{a}}\right] . }
\end{aligned}
$$

The overlap integrals associated with the three remaining diagrams are given in Appendix B, and the detailed evaluation of a decay amplitude $\mathcal{M}_{L S}$ and a decay rate is illustrated in Appendix $\mathrm{C}$ for the decay $\rho \rightarrow \pi \pi$ with a $\mathrm{j}^{0} \mathrm{Kj}^{0}$ interaction. 


\section{JKJ $\mathrm{A} \rightarrow \mathrm{BC}$ matrix elements: explicit results for SHO wavefunctions.}

The complete decay amplitudes for the channels spanned by our representative set of light two-body meson decays are given below. These include decay amplitudes from 1)

linear scalar, 2) constant scalar, 3) Coulomb OGE and 4) transverse OGE terms; a common factor is removed,

$$
\mathcal{M}_{L S}=\frac{\beta^{1 / 2}}{\pi^{3 / 4} m_{q}} e^{-x^{2} / 12} \cdot \tilde{\mathcal{M}}_{L S}
$$

and the transverse OGE amplitude is shown as separate 4) $\delta_{i j}$ and 5) $-Q_{i} Q_{j} / \vec{Q}^{2}$ contributions. These five results for each channel are

$$
\begin{aligned}
& \tilde{\mathcal{M}}_{10}^{\left({ }^{3} \mathrm{~S}_{1} \rightarrow{ }^{1} \mathrm{~S}_{0}+{ }^{1} \mathrm{~S}_{0}\right)}= \\
& -\frac{2^{3} 5}{3^{3}}\left(\frac{b}{\beta^{2}}\right) x\left[{ }_{1} \mathrm{~F}_{1}\left(-\frac{1}{2} ; \frac{3}{2} ; \xi\right)+\frac{4}{45}{ }_{1} \mathrm{~F}_{1}\left(-\frac{1}{2} ; \frac{5}{2} ; \xi\right)\right] \\
& -\frac{2^{9}}{3^{11 / 2}} \pi^{1 / 2}\left(\frac{S_{0}}{\beta}\right) x \\
& +\frac{2^{3}}{3^{3}} \alpha_{s} x\left[{ }_{1} \mathrm{~F}_{1}\left(\frac{1}{2} ; \frac{3}{2} ; \xi\right)-\frac{2}{3}{ }_{1} \mathrm{~F}_{1}\left(\frac{1}{2} ; \frac{5}{2} ; \xi\right)\right] \\
& +\frac{2^{3}}{3^{3}} \alpha_{s} x\left[{ }_{1} \mathrm{~F}_{1}\left(\frac{1}{2} ; \frac{3}{2} ; \xi\right)+\frac{10}{9}{ }_{1} \mathrm{~F}_{1}\left(\frac{1}{2} ; \frac{5}{2} ; \xi\right)\right] \\
& -\frac{2^{3}}{3^{2}} \alpha_{s} x\left[{ }_{1} \mathrm{~F}_{1}\left(\frac{1}{2} ; \frac{3}{2} ; \xi\right)-\frac{2}{3}{ }_{1} \mathrm{~F}_{1}\left(\frac{1}{2} ; \frac{5}{2} ; \xi\right)\right] \text {, } \\
& \tilde{\mathcal{M}}_{20}^{\left({ }^{3} \mathrm{P}_{2} \rightarrow{ }^{1} \mathrm{~S}_{0}+{ }^{1} \mathrm{~S}_{0}\right)}= \\
& +\frac{2^{2} 5^{1 / 2}}{3^{3}}\left(\frac{b}{\beta^{2}}\right) x^{2}\left[{ }_{1} \mathrm{~F}_{1}\left(-\frac{1}{2} ; \frac{3}{2} ; \xi\right)+\frac{8}{15}{ }_{1} \mathrm{~F}_{1}\left(-\frac{1}{2} ; \frac{5}{2} ; \xi\right)+\frac{8}{225}{ }_{1} \mathrm{~F}_{1}\left(-\frac{1}{2} ; \frac{7}{2} ; \xi\right)\right] \\
& +\frac{2^{10}}{3^{13 / 2} 5^{1 / 2}} \pi^{1 / 2}\left(\frac{S_{0}}{\beta}\right) x^{2} \\
& -\frac{2^{2}}{3^{3} 5^{1 / 2}} \alpha_{s} x^{2}\left[{ }_{1} \mathrm{~F}_{1}\left(\frac{1}{2} ; \frac{3}{2} ; \xi\right)-\frac{4}{9}{ }_{1} \mathrm{~F}_{1}\left(\frac{1}{2} ; \frac{5}{2} ; \xi\right)-\frac{8}{45}{ }_{1} \mathrm{~F}_{1}\left(\frac{1}{2} ; \frac{7}{2} ; \xi\right)\right] \\
& -\frac{2^{2}}{3^{3} 5^{1 / 2}} \alpha_{s} x^{2}\left[{ }_{1} \mathrm{~F}_{1}\left(\frac{1}{2} ; \frac{3}{2} ; \xi\right)+\frac{4}{3}{ }_{1} \mathrm{~F}_{1}\left(\frac{1}{2} ; \frac{5}{2} ; \xi\right)+\frac{8}{27}{ }_{1} \mathrm{~F}_{1}\left(\frac{1}{2} ; \frac{7}{2} ; \xi\right)\right] \\
& +\frac{2^{2}}{3^{2} 5^{1 / 2}} \alpha_{s} x^{2}\left[{ }_{1} \mathrm{~F}_{1}\left(\frac{1}{2} ; \frac{3}{2} ; \xi\right)-\frac{8}{27}{ }_{1} \mathrm{~F}_{1}\left(\frac{1}{2} ; \frac{5}{2} ; \xi\right)-\frac{8}{27}{ }_{1} \mathrm{~F}_{1}\left(\frac{1}{2} ; \frac{7}{2} ; \xi\right)\right] \text {, }
\end{aligned}
$$




$$
\tilde{\mathcal{M}}_{21}^{\left({ }^{3} \mathrm{P}_{2} \rightarrow{ }^{3} \mathrm{~S}_{1}+{ }^{1} \mathrm{~S}_{0}\right)}=
$$

$$
\begin{aligned}
& -\frac{2^{3 / 2} 5^{1 / 2}}{3^{5 / 2}}\left(\frac{b}{\beta^{2}}\right) x^{2}\left[{ }_{1} \mathrm{~F}_{1}\left(-\frac{1}{2} ; \frac{3}{2} ; \xi\right)+\frac{8}{15} \mathrm{~F}_{1}\left(-\frac{1}{2} ; \frac{5}{2} ; \xi\right)+\frac{8}{225}{ }_{1} \mathrm{~F}_{1}\left(-\frac{1}{2} ; \frac{7}{2} ; \xi\right)\right] \\
& -\frac{2^{19 / 2}}{3^{6} 5^{1 / 2}} \pi^{1 / 2}\left(\frac{S_{0}}{\beta}\right) x^{2} \\
& +\frac{2^{3 / 2}}{3^{5 / 2} 5^{1 / 2}} \alpha_{s} x^{2}\left[{ }_{1} \mathrm{~F}_{1}\left(\frac{1}{2} ; \frac{3}{2} ; \xi\right)-\frac{4}{9} \mathrm{~F}_{1}\left(\frac{1}{2} ; \frac{5}{2} ; \xi\right)-\frac{8}{45}{ }_{1} \mathrm{~F}_{1}\left(\frac{1}{2} ; \frac{7}{2} ; \xi\right)\right] \\
& +\frac{2^{5 / 2}}{3^{5 / 2} 5^{1 / 2}} \alpha_{s} x^{2}\left[{ }_{1} \mathrm{~F}_{1}\left(\frac{1}{2} ; \frac{3}{2} ; \xi\right)+\frac{4}{9} \mathrm{~F}_{1} \mathrm{~F}_{1}\left(\frac{1}{2} ; \frac{5}{2} ; \xi\right)+\frac{8}{135}{ }_{1} \mathrm{~F}_{1}\left(\frac{1}{2} ; \frac{7}{2} ; \xi\right)\right] \\
& -\frac{2^{3 / 2}}{3^{3 / 2} 5^{1 / 2}} \alpha_{s} x^{2}\left[{ }_{1} \mathrm{~F}_{1}\left(\frac{1}{2} ; \frac{3}{2} ; \xi\right)-\frac{8}{27}{ }_{1} \mathrm{~F}_{1}\left(\frac{1}{2} ; \frac{5}{2} ; \xi\right)-\frac{8}{27}{ }_{1} \mathrm{~F}_{1}\left(\frac{1}{2} ; \frac{7}{2} ; \xi\right)\right]
\end{aligned}
$$

$\tilde{\mathcal{M}}_{01}^{\left({ }^{3} \mathrm{P}_{1} \rightarrow{ }^{3} \mathrm{~S}_{1}+{ }^{1} \mathrm{~S}_{0}\right)}=$

$$
\begin{aligned}
& -\frac{2^{5} 5}{3^{7 / 2}}\left(\frac{b}{\beta^{2}}\right)\left[{ }_{1} \mathrm{~F}_{1}\left(-\frac{3}{2} ;-\frac{1}{2} ; \xi\right)-\frac{12}{5}{ }_{1} \mathrm{~F}_{1}\left(-\frac{3}{2} ; \frac{1}{2} ; \xi\right)+\frac{8}{15}{ }_{1} \mathrm{~F}_{1}\left(-\frac{3}{2} ; \frac{3}{2} ; \xi\right)\right] \\
& +\frac{2^{9}}{3^{5}} \pi^{1 / 2}\left(\frac{S_{0}}{\beta}\right)\left(1-\frac{2}{9} x^{2}\right) \\
& +\frac{2^{5}}{3^{5 / 2}} \alpha_{s}\left[{ }_{1} \mathrm{~F}_{1}\left(-\frac{1}{2} ;-\frac{1}{2} ; \xi\right)+\frac{4}{3}{ }_{1} \mathrm{~F}_{1}\left(-\frac{1}{2} ; \frac{1}{2} ; \xi\right)-\frac{8}{3}{ }_{1} \mathrm{~F}_{1}\left(-\frac{1}{2} ; \frac{3}{2} ; \xi\right)\right] \\
& +\frac{2^{6}}{3^{5 / 2}} \alpha_{s}\left[{ }_{1} \mathrm{~F}_{1}\left(-\frac{1}{2} ;-\frac{1}{2} ; \xi\right)-\frac{10}{3}{ }_{1} \mathrm{~F}_{1}\left(-\frac{1}{2} ; \frac{1}{2} ; \xi\right)+\frac{28}{9}{ }_{1} \mathrm{~F}_{1}\left(-\frac{1}{2} ; \frac{3}{2} ; \xi\right)\right] \\
& -\frac{2^{5}}{3^{3 / 2}} \alpha_{s}\left[{ }_{1} \mathrm{~F}_{1}\left(-\frac{1}{2} ;-\frac{1}{2} ; \xi\right)+\frac{8}{9}{ }_{1} \mathrm{~F}_{1}\left(-\frac{1}{2} ; \frac{1}{2} ; \xi\right)-\frac{16}{9}{ }_{1} \mathrm{~F}_{1}\left(-\frac{1}{2} ; \frac{3}{2} ; \xi\right)\right]
\end{aligned}
$$

$$
\begin{aligned}
\tilde{\mathcal{M}}_{21}{ }^{\left.3 \mathrm{P}_{1} \rightarrow{ }^{3} \mathrm{~S}_{1}+{ }^{1} \mathrm{~S}_{0}\right)}= & \\
& -\frac{2^{3 / 2} 5}{3^{7 / 2}}\left(\frac{b}{\beta^{2}}\right) x^{2}\left[{ }_{1} \mathrm{~F}_{1}\left(-\frac{1}{2} ; \frac{3}{2} ; \xi\right)+\frac{8}{15}{ }_{1} \mathrm{~F}_{1}\left(-\frac{1}{2} ; \frac{5}{2} ; \xi\right)+\frac{8}{225}{ }_{1} \mathrm{~F}_{1}\left(-\frac{1}{2} ; \frac{7}{2} ; \xi\right)\right] \\
& -\frac{2^{19 / 2}}{3^{7}} \pi^{1 / 2}\left(\frac{S_{0}}{\beta}\right) x^{2} \\
& +\frac{2^{3 / 2}}{3^{7 / 2}} \alpha_{s} x^{2}\left[{ }_{1} \mathrm{~F}_{1}\left(\frac{1}{2} ; \frac{3}{2} ; \xi\right)-\frac{4}{9}{ }_{1} \mathrm{~F}_{1}\left(\frac{1}{2} ; \frac{5}{2} ; \xi\right)-\frac{8}{45}{ }_{1} \mathrm{~F}_{1}\left(\frac{1}{2} ; \frac{7}{2} ; \xi\right)\right]
\end{aligned}
$$




$$
\begin{aligned}
& +\frac{2^{5 / 2}}{3^{7 / 2}} \alpha_{s} x^{2}\left[{ }_{1} \mathrm{~F}_{1}\left(\frac{1}{2} ; \frac{3}{2} ; \xi\right)+\frac{4}{9}{ }_{1} \mathrm{~F}_{1}\left(\frac{1}{2} ; \frac{5}{2} ; \xi\right)+\frac{8}{135}{ }_{1} \mathrm{~F}_{1}\left(\frac{1}{2} ; \frac{7}{2} ; \xi\right)\right] \\
& -\frac{2^{3 / 2}}{3^{5 / 2}} \alpha_{s} x^{2}\left[{ }_{1} \mathrm{~F}_{1}\left(\frac{1}{2} ; \frac{3}{2} ; \xi\right)-\frac{8}{27}{ }_{1} \mathrm{~F}_{1}\left(\frac{1}{2} ; \frac{5}{2} ; \xi\right)-\frac{8}{27}{ }_{1} \mathrm{~F}_{1}\left(\frac{1}{2} ; \frac{7}{2} ; \xi\right)\right]
\end{aligned}
$$

$$
\begin{aligned}
\tilde{\mathcal{M}}_{00}^{\left({ }^{3} \mathrm{P}_{0} \rightarrow{ }^{1} \mathrm{~S}_{0}+{ }^{1} \mathrm{~S}_{0}\right)}= & \\
& -\frac{2^{9 / 2}}{3^{3}}\left(\frac{b}{\beta^{2}}\right)\left[{ }_{1} \mathrm{~F}_{1}\left(-\frac{3}{2} ;-\frac{1}{2} ; \xi\right)-\frac{12}{5}{ }_{1} \mathrm{~F}_{1}\left(-\frac{3}{2} ; \frac{1}{2} ; \xi\right)+\frac{8}{15}{ }_{1} \mathrm{~F}_{1}\left(-\frac{3}{2} ; \frac{3}{2} ; \xi\right)\right] \\
& +\frac{2^{17 / 2}}{3^{9 / 2}} \pi^{1 / 2}\left(\frac{S_{0}}{\beta}\right)\left(1-\frac{2}{9} x^{2}\right) \\
& +\frac{2^{9 / 2}}{3^{2}} \alpha_{s}\left[{ }_{1} \mathrm{~F}_{1}\left(-\frac{1}{2} ;-\frac{1}{2} ; \xi\right)+\frac{4}{3}{ }_{1} \mathrm{~F}_{1}\left(-\frac{1}{2} ; \frac{1}{2} ; \xi\right)-\frac{8}{3}{ }_{1} \mathrm{~F}_{1}\left(-\frac{1}{2} ; \frac{3}{2} ; \xi\right)\right] \\
& +\frac{2^{9 / 2}}{3^{2}} \alpha_{s}\left[{ }_{1} \mathrm{~F}_{1}\left(-\frac{1}{2} ;-\frac{1}{2} ; \xi\right)-8{ }_{1} \mathrm{~F}_{1}\left(-\frac{1}{2} ; \frac{1}{2} ; \xi\right)+\frac{80}{9}{ }_{1} \mathrm{~F}_{1}\left(-\frac{1}{2} ; \frac{3}{2} ; \xi\right)\right] \\
& -\frac{2^{9 / 2}}{3} \alpha_{s}\left[{ }_{1} \mathrm{~F}_{1}\left(-\frac{1}{2} ;-\frac{1}{2} ; \xi\right)+\frac{8}{9}{ }_{1} \mathrm{~F}_{1}\left(-\frac{1}{2} ; \frac{1}{2} ; \xi\right)-\frac{16}{9}{ }_{1} \mathrm{~F}_{1}\left(-\frac{1}{2} ; \frac{3}{2} ; \xi\right)\right]
\end{aligned}
$$

$\tilde{\mathcal{M}}_{01}^{\left({ }^{1} \mathrm{P}_{1} \rightarrow{ }^{3} \mathrm{~S}_{1}+{ }^{1} \mathrm{~S}_{0}\right)}=$

$$
\begin{aligned}
& +\frac{2^{9 / 2} 5}{3^{7 / 2}}\left(\frac{b}{\beta^{2}}\right)\left[{ }_{1} \mathrm{~F}_{1}\left(-\frac{3}{2} ;-\frac{1}{2} ; \xi\right)-\frac{12}{5}{ }_{1} \mathrm{~F}_{1}\left(-\frac{3}{2} ; \frac{1}{2} ; \xi\right)+\frac{8}{15}{ }_{1} \mathrm{~F}_{1}\left(-\frac{3}{2} ; \frac{3}{2} ; \xi\right)\right] \\
& -\frac{2^{17 / 2}}{3^{5}} \pi^{1 / 2}\left(\frac{S_{0}}{\beta}\right)\left(1-\frac{2}{9} x^{2}\right) \\
& -\frac{2^{9 / 2}}{3^{5 / 2}} \alpha_{s}\left[{ }_{1} \mathrm{~F}_{1}\left(-\frac{1}{2} ;-\frac{1}{2} ; \xi\right)+\frac{4}{3}{ }_{1} \mathrm{~F}_{1}\left(-\frac{1}{2} ; \frac{1}{2} ; \xi\right)-\frac{8}{3}{ }_{1} \mathrm{~F}_{1}\left(-\frac{1}{2} ; \frac{3}{2} ; \xi\right)\right] \\
& -\frac{2^{9 / 2}}{3^{3 / 2}} \alpha_{s}\left[{ }_{1} \mathrm{~F}_{1}\left(-\frac{1}{2} ;-\frac{1}{2} ; \xi\right)-\frac{16}{9}{ }_{1} \mathrm{~F}_{1}\left(-\frac{1}{2} ; \frac{1}{2} ; \xi\right)+\frac{32}{27}{ }_{1} \mathrm{~F}_{1}\left(-\frac{1}{2} ; \frac{3}{2} ; \xi\right)\right] \\
& +\frac{2^{9 / 2}}{3^{3 / 2}} \alpha_{s}\left[{ }_{1} \mathrm{~F}_{1}\left(-\frac{1}{2} ;-\frac{1}{2} ; \xi\right)+\frac{8}{9}{ }_{1} \mathrm{~F}_{1}\left(-\frac{1}{2} ; \frac{1}{2} ; \xi\right)-\frac{16}{9}{ }_{1} \mathrm{~F}_{1}\left(-\frac{1}{2} ; \frac{3}{2} ; \xi\right)\right],
\end{aligned}
$$

$$
\begin{aligned}
\tilde{\mathcal{M}}_{21}^{\left({ }^{1} \mathrm{P}_{1} \rightarrow{ }^{3} \mathrm{~S}_{1}+{ }^{1} \mathrm{~S}_{0}\right)=} & \\
& -\frac{2^{2} 5}{3^{7 / 2}}\left(\frac{b}{\beta^{2}}\right) x^{2}\left[{ }_{1} \mathrm{~F}_{1}\left(-\frac{1}{2} ; \frac{3}{2} ; \xi\right)+\frac{8}{15}{ }_{1} \mathrm{~F}_{1}\left(-\frac{1}{2} ; \frac{5}{2} ; \xi\right)+\frac{8}{225}{ }_{1} \mathrm{~F}_{1}\left(-\frac{1}{2} ; \frac{7}{2} ; \xi\right)\right]
\end{aligned}
$$




$$
\begin{aligned}
& -\frac{2^{10}}{3^{7}} \pi^{1 / 2}\left(\frac{S_{0}}{\beta}\right) x^{2} \\
& +\frac{2^{2}}{3^{7 / 2}} \alpha_{s} x^{2}\left[{ }_{1} \mathrm{~F}_{1}\left(\frac{1}{2} ; \frac{3}{2} ; \xi\right)-\frac{4}{9}{ }_{1} \mathrm{~F}_{1}\left(\frac{1}{2} ; \frac{5}{2} ; \xi\right)-\frac{8}{45}{ }_{1} \mathrm{~F}_{1}\left(\frac{1}{2} ; \frac{7}{2} ; \xi\right)\right] \\
& +\frac{2^{2}}{3^{5 / 2}} \alpha_{s} x^{2}\left[{ }_{1} \mathrm{~F}_{1}\left(\frac{1}{2} ; \frac{3}{2} ; \xi\right)+\frac{4}{27}{ }_{1} \mathrm{~F}_{1}\left(\frac{1}{2} ; \frac{5}{2} ; \xi\right)-\frac{8}{405}{ }_{1} \mathrm{~F}_{1}\left(\frac{1}{2} ; \frac{7}{2} ; \xi\right)\right] \\
& -\frac{2^{2}}{3^{5 / 2}} \alpha_{s} x^{2}\left[{ }_{1} \mathrm{~F}_{1}\left(\frac{1}{2} ; \frac{3}{2} ; \xi\right)-\frac{8}{27}{ }_{1} \mathrm{~F}_{1}\left(\frac{1}{2} ; \frac{5}{2} ; \xi\right)-\frac{8}{27}{ }_{1} \mathrm{~F}_{1}\left(\frac{1}{2} ; \frac{7}{2} ; \xi\right)\right] .
\end{aligned}
$$

\section{JKJ decay rates: numerical results.}

To estimate the numerical importance of the scalar and OGE decay mechanisms we first

specialize to $\rho \rightarrow \pi \pi$ and consider each contribution in isolation. Using (6), (40) and (41),

and the flavor factors in App.A, the decay rate due to linear scalar pair production alone would be

$$
\Gamma_{\rho \rightarrow \pi \pi}^{\mathrm{sKs}}=\pi^{-1 / 2}\left(\frac{2^{6} 5^{2}}{3^{6}}\right)\left(\frac{b}{m_{q} \beta}\right)^{2} \frac{E_{\pi}^{2}}{M_{\rho}} x^{3}\left[{ }_{1} \mathrm{~F}_{1}\left(-\frac{1}{2} ; \frac{3}{2} ; \xi\right)+\frac{4}{45}{ }_{1} \mathrm{~F}_{1}\left(-\frac{1}{2} ; \frac{5}{2} ; \xi\right)\right]^{2} e^{-x^{2} / 6}
$$

The analogous decay rates due to Coulomb OGE and transverse OGE are

$$
\Gamma_{\rho \rightarrow \pi \pi}^{\mathrm{j}^{0} \mathrm{Kj}{ }^{0}}=\pi^{-1 / 2}\left(\frac{2^{6}}{3^{6}}\right) \alpha_{s}^{2}\left(\frac{\beta}{m_{q}}\right)^{2} \frac{E_{\pi}^{2}}{M_{\rho}} x^{3}\left[{ }_{1} \mathrm{~F}_{1}\left(\frac{1}{2} ; \frac{3}{2} ; \xi\right)-\frac{2}{3}{ }_{1} \mathrm{~F}_{1}\left(\frac{1}{2} ; \frac{5}{2} ; \xi\right)\right]^{2} e^{-x^{2} / 6}
$$

and

$$
\Gamma_{\rho \rightarrow \pi \pi}^{\mathrm{j}^{\mathrm{T}} \mathrm{Kj}^{\mathrm{T}}}=\pi^{-1 / 2}\left(\frac{2^{8}}{3^{6}}\right) \alpha_{s}^{2}\left(\frac{\beta}{m_{q}}\right)^{2} \frac{E_{\pi}^{2}}{M_{\rho}} x^{3}\left[{ }_{1} \mathrm{~F}_{1}\left(\frac{1}{2} ; \frac{3}{2} ; \xi\right)+\frac{4}{9}{ }_{1} \mathrm{~F}_{1}\left(\frac{1}{2} ; \frac{5}{2} ; \xi\right)\right]^{2} e^{-x^{2} / 6}
$$

These rates are shown in figure 5 for the parameter set $\alpha_{s}=0.6, b=0.18 \mathrm{GeV}^{2}$ and $m_{q}=0.33 \mathrm{GeV}$. Evidently the dominant decay mechanism is the sKs interaction, pair production through the scalar confining potential. At $\beta=0.4 \mathrm{GeV}$ the $\rho \rightarrow \pi \pi$ width predicted by the sKs model alone is $330 \mathrm{MeV}$, about twice the experimental $151 \mathrm{MeV}$. In comparison, transverse OGE gives a width of $3.9 \mathrm{MeV}$, and the color Coulomb interaction gives only 0.36 MeV. (The transverse and Coulomb OGE contributions add constructively, so the total width from OGE alone would be $6.7 \mathrm{MeV}$. These both interfere destructively 
with the dominant sKs amplitude, so the total width we find from all three amplitudes is $\left.\Gamma_{\rho \rightarrow \pi \pi}=243 \mathrm{MeV}.\right)$

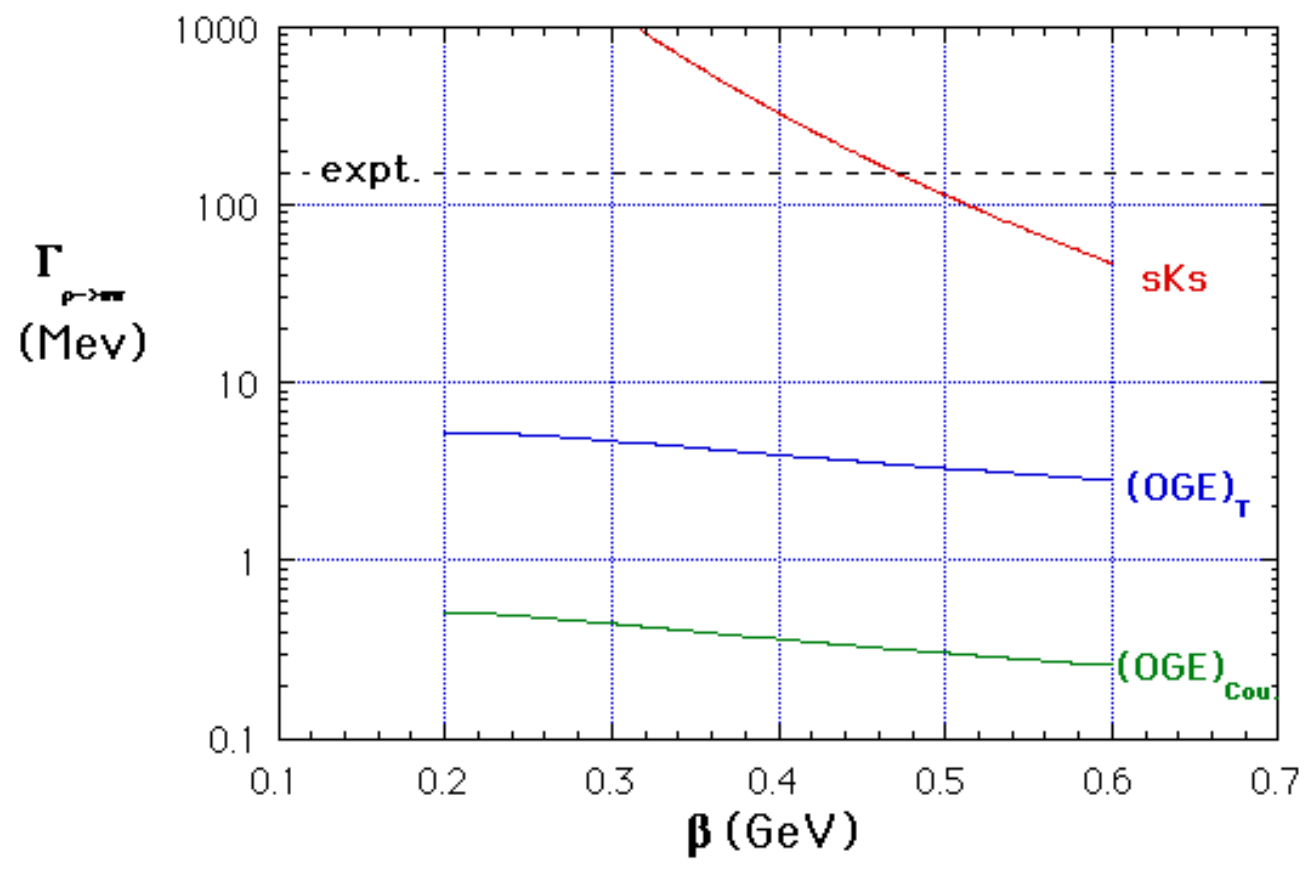

Figure 5. The decay rate $\Gamma_{\rho \rightarrow \pi \pi}$ assuming only $\mathrm{sKs}, \mathrm{j}^{\mathrm{T}} \mathrm{Kj}^{\mathrm{T}}$ or $\mathrm{j}^{0} \mathrm{Kj}^{0}$ decay interactions. Parameters $\alpha_{s}=0.6, b=0.18 \mathrm{GeV}^{2}, m_{q}=0.33 \mathrm{GeV}$.

Although the sKs interaction is usually found to be dominant in our representative set of decays, the OGE contributions are often comparable to sKs and cannot generally be ignored. To illustrate this, in figure 6 we show the numerical decay amplitudes we find in each decay. The normalization incorporates phase space, so these amplitudes squared give the physical decay rates. The magnitude of the experimental amplitude is indicated by horizontal lines. (The $h_{1}$ amplitudes are not shown because they are essentially identical to the $b_{1}$ amplitudes times a flavor factor of $\sqrt{3}$.) 
a) $\rho \rightarrow \pi \pi$

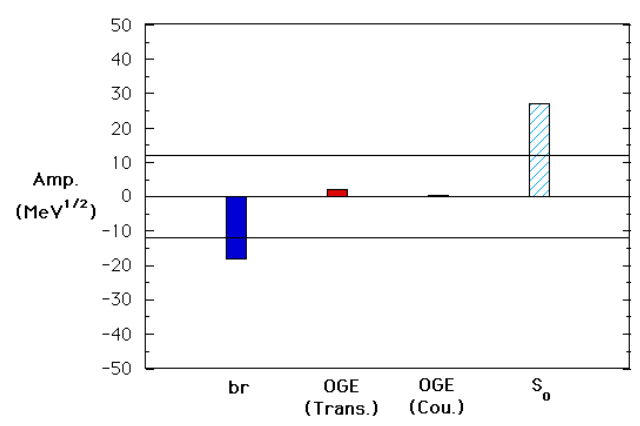

c) $a_{2}->\rho \pi$

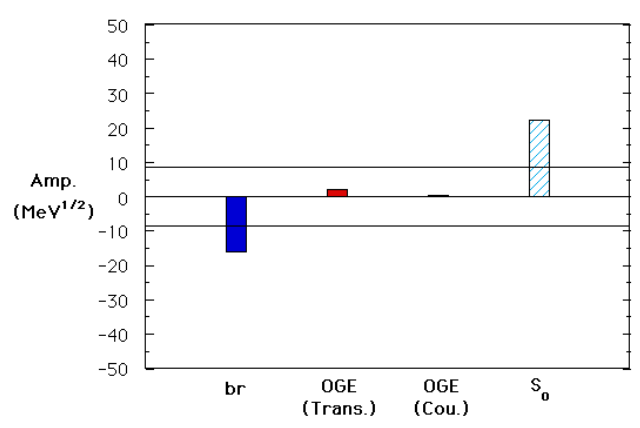

d) $a_{1}->(\rho \pi)_{s}$

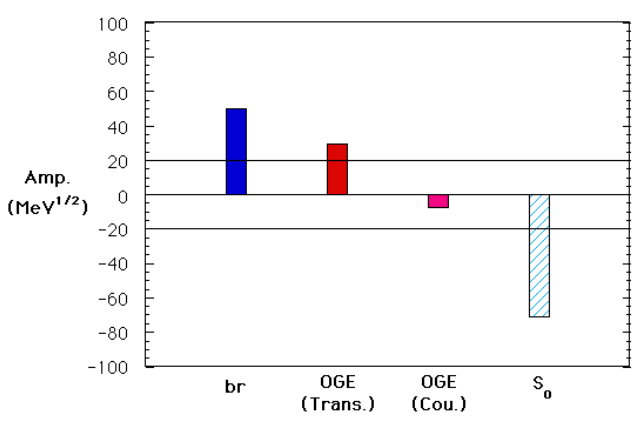

f) $b_{1} \rightarrow(\omega \pi)_{s}$

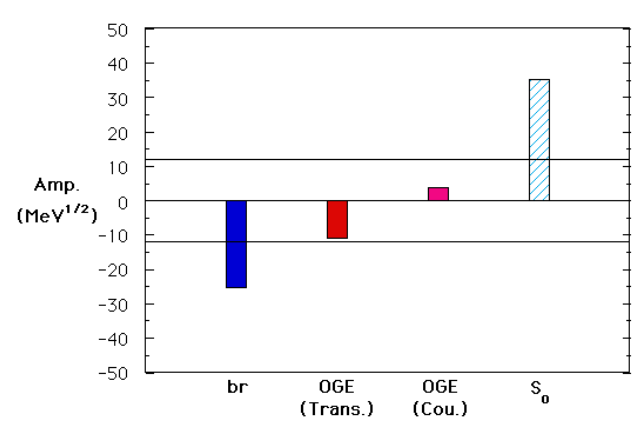

b) $f_{2} \rightarrow \pi \pi$

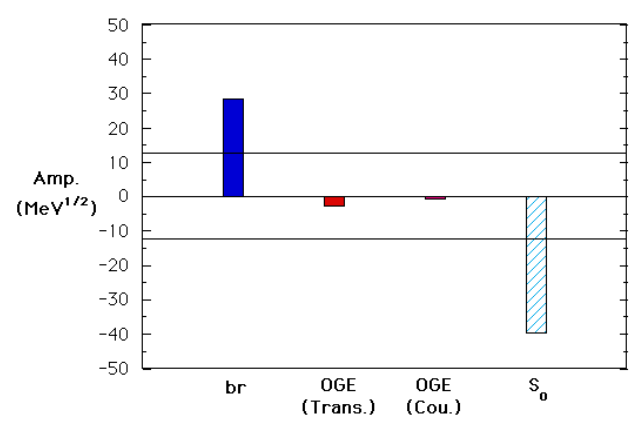

e) $a_{1}->(\rho \pi)$

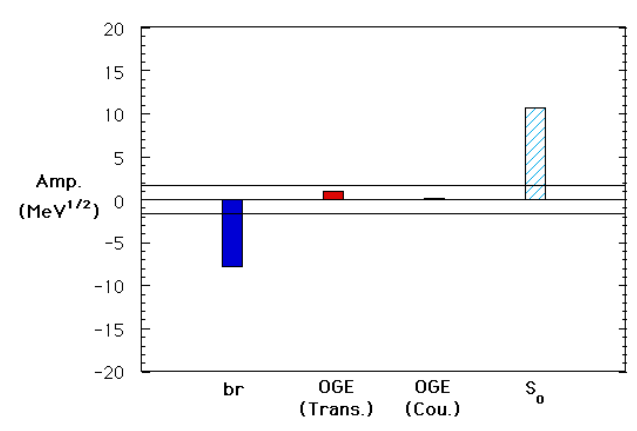

g) $b_{1} \rightarrow(\omega \pi)_{D}$

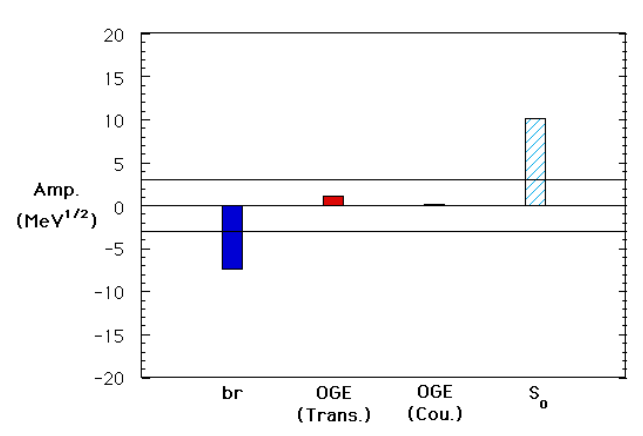



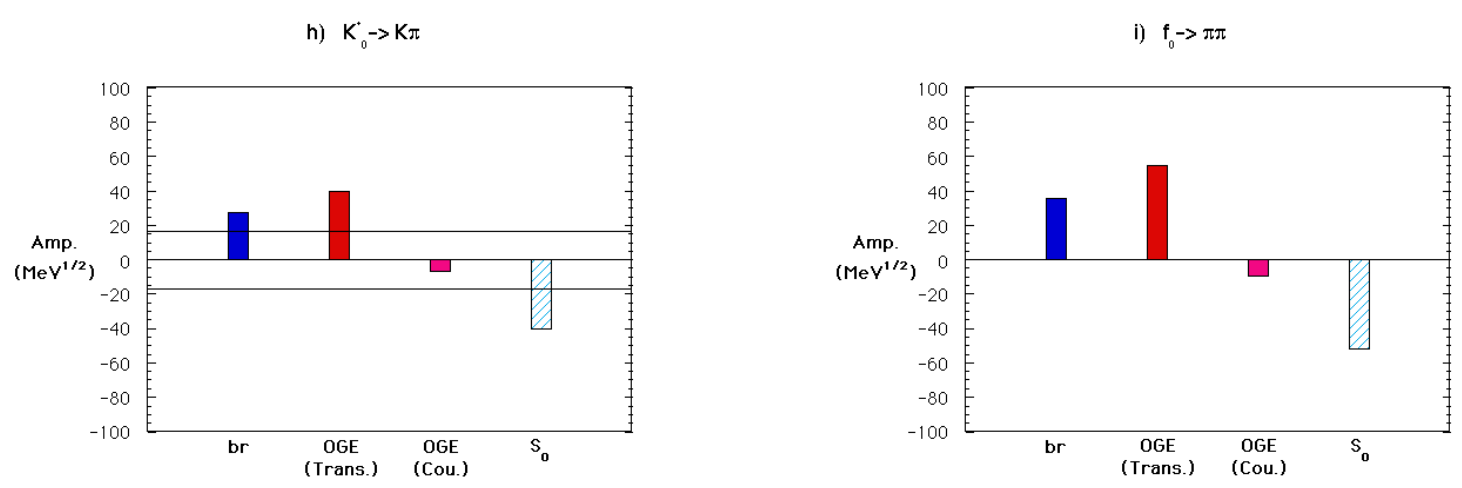

Figure 6a-i. Decay amplitudes from sKs (br only), OGE (transverse) and OGE (coulomb) decay mechanisms, with parameters $\beta=0.4 \mathrm{GeV}, b=0.18 \mathrm{GeV}^{2}, \alpha_{s}=0.6$ and $m_{q}=$ $0.33 \mathrm{GeV}$. The decay amplitude from a constant scalar ${ }^{3} \mathrm{P}_{0}$ term of $S_{0}=-0.5 \mathrm{GeV}$ is shown for comparison.

The decay amplitudes due to Coulomb and transverse OGE are also shown in the histograms. The Coulomb gluon term is small in all the decays we have considered. This could have been anticipated; in the limit of a constant kernel $K$ the Coulomb gluon decay amplitude actually vanishes, because the transition operator is then proportional to the fermion number operator $Q=\int d^{3} x j^{0}$ (squared) and hence cannot pair produce. With a slowly varying kernel the Coulomb decay amplitude is nonzero, but remains small. Transverse gluon exchange in contrast is sufficiently large to make an important contribution in some channels. The most notable of these are $\mathrm{K}_{0}^{*} \rightarrow \mathrm{K} \pi$ and $f_{0} \rightarrow \pi \pi$, in which the OGE decay amplitude actually dominates the nonperturbative sKs amplitude (see figures $6 h, i$ ). Recall that the widths of scalar $q \bar{q}$ states are problematical in the ${ }^{3} \mathrm{P}_{0}$ model because of a node in their decay amplitudes (see figure 2). The large additional OGE decay amplitude insures that the scalar $q \bar{q}$ states will be broad resonances, despite the node in the sKs or ${ }^{3} \mathrm{P}_{0}$ decay amplitudes.

Note that the overall scale of the total decay amplitudes predicted by the sKs+OGE decay mechanisms is too large relative to data by about a factor of two. It is nontrivial that 
there is even approximate agreement; recall in contrast that the overall scale of the decay rates is not determined in the ${ }^{3} \mathrm{P}_{0}$ model, but is fitted using the parameter $\gamma$. Since we regard the values $\alpha_{s}=0.6, b=0.18 \mathrm{GeV}^{2}$ and $m_{q}=0.33 \mathrm{GeV}$ as reasonably well established for light quarks, we doubt that the discrepancy reflects our choices for these parameters. For this reason we do not show an optimized fit to the experimental rates, which would give reduced values for $\alpha_{s}$ or $b$.

We suggest that the theoretical overestimate of scale may indicate the presence of important relativistic corrections to our nonrelativistic amplitudes, such as $m / E$ factors in (38); if included these would reduce the overall scale of rates and have little effect on the successful relative rate predictions. Other possibile sources of this discrepancy include our choice of $q \bar{q}$ wavefunctions (the overall scale of rates is very sensitive to the SHO width parameter $\beta$ ) and our assumption that confinement may be treated as the exchange of a scalar quantum with a linear kernel in configuration space but no $q$-q-scalar form factor.

A large negative constant $S_{0}$ of up to $\approx-1 \mathrm{GeV}$ is often included in potential models, and is needed to give a best fit to spectroscopy. Of course it may not actually be present in the scalar potential $S(r)$, and may instead simply be a way of subtracting off a fictitious constituent quark mass contribution of $2 m_{q} \approx 0.7 \mathrm{GeV}$ from the $q \bar{q}$ spectrum. This constant would contribute a decay amplitude identical to a ${ }^{3} \mathrm{P}_{0}$ coupling of $\gamma=\left(2^{4} / 3^{5 / 2}\right) S_{0} / m_{q}$; for completeness we have included such a constant term in our amplitude histograms, with an intermediate magnitude of $S_{0}=-0.5 \mathrm{GeV}$. If an $S_{0}$ of approximately this magnitude were actually present it clearly would make an important contribution, as it is larger than the $b r$ term and opposite in sign. We find however that any $S_{0}$ of comparable magnitude leads to unrealistic $\mathrm{D} / \mathrm{S}$ ratios in $b_{1} \rightarrow \omega \pi$ and $a_{1} \rightarrow \rho \pi$. In both decays $\mathrm{D} / \mathrm{S}$ passes through zero near $S_{0}=-0.3 \mathrm{GeV}$ and diverges near $S_{0}=-0.5 \mathrm{GeV}$ with conventional parameter values of $\alpha_{s}=0.6, \beta=0.4 \mathrm{GeV}, b=0.18 \mathrm{GeV}^{2}$ and $m_{q}=0.33 \mathrm{GeV}$, whereas $S_{0}=0$ leads to reasonable $\mathrm{D} / \mathrm{S}$ values. Since the $\mathrm{D} / \mathrm{S}$ ratios are inconsistent with a large negative constant $S_{0}$ we will assume $S_{0}=0$ subsequently. 


\section{E. D/S ratios in JKJ decay models}

In Sec.I.C we noted that the ratio of S- and D-wave amplitudes in the decays $b_{1} \rightarrow \omega \pi$ and $a_{1} \rightarrow \rho \pi$ allowed sensitive tests of the angular quantum numbers of the $q \bar{q}$ pair produced in a strong decay, and that the ${ }^{3} \mathrm{P}_{0}$ model is in reasonable agreement with experiment, albeit for the rather large value of $\beta=0.45 \mathrm{GeV}$. Recall also that the ratio of $\mathrm{D} / \mathrm{S}$ ratios $\left(a_{1} / b_{1}\right)$ in the ${ }^{3} \mathrm{P}_{0}$ model is predicted to be $-1 / 2$ (neglecting minor phase space differences), independent of the $\mathrm{L}_{q \bar{q}}=0$ and $\mathrm{L}_{q \bar{q}}=1$ radial wavefunctions.

We find that the sKs interaction leads to very similar predictions to the ${ }^{3} \mathrm{P}_{0}$ model. The sKs predictions for D/S ratios can be read from the decay amplitudes (41-48); for $b_{1} \rightarrow \omega \pi$ it is

$$
\left.\frac{a_{D}}{a_{S}}\right|_{b_{1} \rightarrow \omega \pi} ^{\mathrm{SKs}}=-\frac{x^{2}}{2^{5 / 2}} \frac{\left[{ }_{1} \mathrm{~F}_{1}\left(-\frac{1}{2} ; \frac{3}{2} ; \xi\right)+\frac{8}{15}{ }_{1} \mathrm{~F}_{1}\left(-\frac{1}{2} ; \frac{5}{2} ; \xi\right)+\frac{8}{225}{ }_{1} \mathrm{~F}_{1}\left(-\frac{1}{2} ; \frac{7}{2} ; \xi\right)\right]}{\left[{ }_{1} \mathrm{~F}_{1}\left(-\frac{3}{2} ;-\frac{1}{2} ; \xi\right)-\frac{12}{5}{ }_{1} \mathrm{~F}_{1}\left(-\frac{3}{2} ; \frac{1}{2} ; \xi\right)+\frac{8}{15}{ }_{1} \mathrm{~F}_{1}\left(-\frac{3}{2} ; \frac{3}{2} ; \xi\right)\right]}
$$

This rather complicated result is shown as a function of $\beta$ in figure 7 , together with the ${ }^{3} \mathrm{P}_{0}$ prediction (21) and the corresponding $a_{1} \rightarrow \rho \pi$ results. The sKs and ${ }^{3} \mathrm{P}_{0}$ decay models evidently give remarkably similar D/S ratios, and are essentially indistinguishable in these reactions.

The ratio of $a_{1} / b_{1} \mathrm{D} / \mathrm{S}$ ratios with the sKs interaction is predicted to be

$$
\frac{\left.\frac{a_{D}}{a_{S}}\right|_{a_{1} \rightarrow \rho \pi}}{\left.\frac{a_{D}}{a_{S}}\right|_{b_{1} \rightarrow \omega \pi}}=-\frac{1}{2}
$$

just as in the ${ }^{3} \mathrm{P}_{0}$ model. Thus, to the extent that the ${ }^{3} \mathrm{P}_{0}$ model is successful in explaining $\mathrm{D} / \mathrm{S}$ ratios, this is a success of the sKs model as well. Some similarity could have been anticipated since both the ${ }^{3} \mathrm{P}_{0}$ and sKs decay models assume pair production with ${ }^{3} \mathrm{P}_{0}$ quantum numbers, and differ only in the presence or absence of spatial correlations with incoming quark lines. 


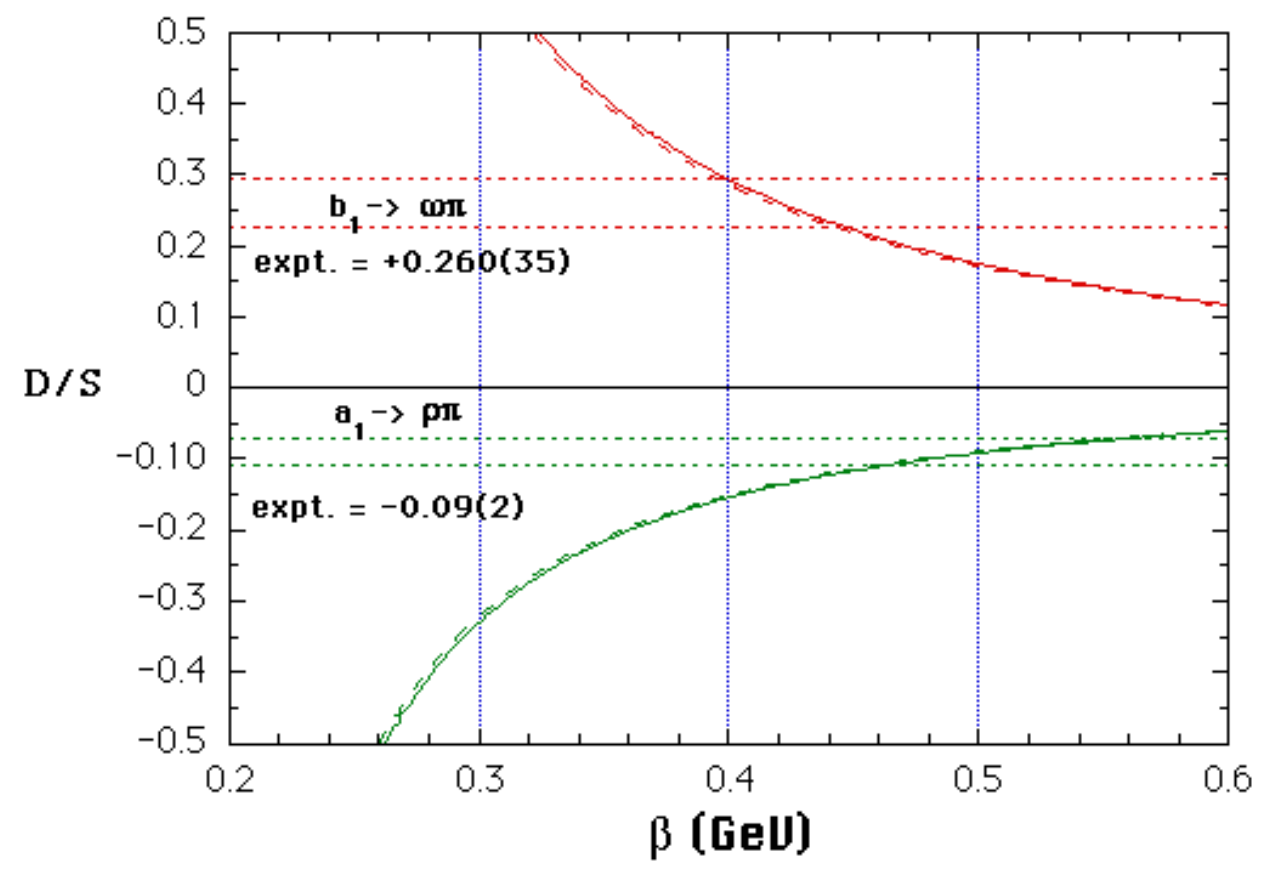

Figure 7. D/S ratios in $b_{1} \rightarrow \omega \pi$ and $a_{1} \rightarrow \rho \pi$ predicted by the ${ }^{3} \mathrm{P}_{0}$ model (dashed) and the sKs model with $S(r)=b r$ (solid).

The simple ratio in (53) follows in the $\mathrm{j}^{0} \mathrm{Kj}{ }^{0}$ model as well, and appears to be generally true if the initial lines have no spin-flip amplitude and identical $\left(a_{1}, b_{1}\right)$ and $(\rho, \omega, \pi)$ radial wavefunctions are assumed. In contrast, the $\mathrm{j}^{\mathrm{T}} \mathrm{Kj}^{\mathrm{T}}$ transverse OGE interaction has an initial-line spin-flip amplitude, so that the ratio departs from $-1 / 2$. This may be useful as a signature of the OGE component in the physical decay amplitude.

Although these D/S ratios are often cited as an argument against an OGE decay mechanism, the actual OGE calculation does not appear to have been carried out in the literature. (We emphasize that OGE is not equivalent to the " ${ }^{3} \mathrm{~S}_{1}$ " decay model, for which results do exist [6.10].) We find that the full (Coulomb + transverse) OGE D/S ratios are given by 


$$
\left.\frac{a_{D}}{a_{S}}\right|_{b_{1} \rightarrow \omega \pi} ^{\mathrm{OGE}}=-\frac{x^{2}}{2^{5 / 2} 3} \frac{\left[{ }_{1} \mathrm{~F}_{1}\left(\frac{1}{2} ; \frac{3}{2} ; \xi\right)+\frac{8}{9}{ }_{1} \mathrm{~F}_{1}\left(\frac{1}{2} ; \frac{5}{2} ; \xi\right)+\frac{88}{135}{ }_{1} \mathrm{~F}_{1}\left(\frac{1}{2} ; \frac{7}{2} ; \xi\right)\right]}{\left[{ }_{1} \mathrm{~F}_{1}\left(-\frac{1}{2} ;-\frac{1}{2} ; \xi\right)-\frac{20}{3}{ }_{1} \mathrm{~F}_{1}\left(-\frac{1}{2} ; \frac{1}{2} ; \xi\right)+\frac{56}{9}{ }_{1} \mathrm{~F}_{1}\left(-\frac{1}{2} ; \frac{3}{2} ; \xi\right)\right]}
$$

and

$$
\left.\frac{a_{D}}{a_{S}}\right|_{a_{1} \rightarrow \rho \pi} ^{\mathrm{OGE}}=-\frac{x^{2}}{2^{9 / 2} 3^{2}} \frac{\left[{ }_{1} \mathrm{~F}_{1}\left(\frac{1}{2} ; \frac{5}{2} ; \xi\right)+\frac{28}{45}{ }_{1} \mathrm{~F}_{1}\left(\frac{1}{2} ; \frac{7}{2} ; \xi\right)\right]}{\left[{ }_{1} \mathrm{~F}_{1}\left(-\frac{1}{2} ; \frac{1}{2} ; \xi\right)-\frac{10}{9}{ }_{1} \mathrm{~F}_{1}\left(-\frac{1}{2} ; \frac{3}{2} ; \xi\right)\right]} .
$$

These are shown in figure 8; evidently the hypothesis of OGE dominance of these decays can indeed be rejected, because the OGE decay mechanism predicts $\mathrm{D} / \mathrm{S}$ amplitude ratios of the wrong sign for both $a_{1} \rightarrow \rho \pi$ and $b_{1} \rightarrow \omega \pi$.

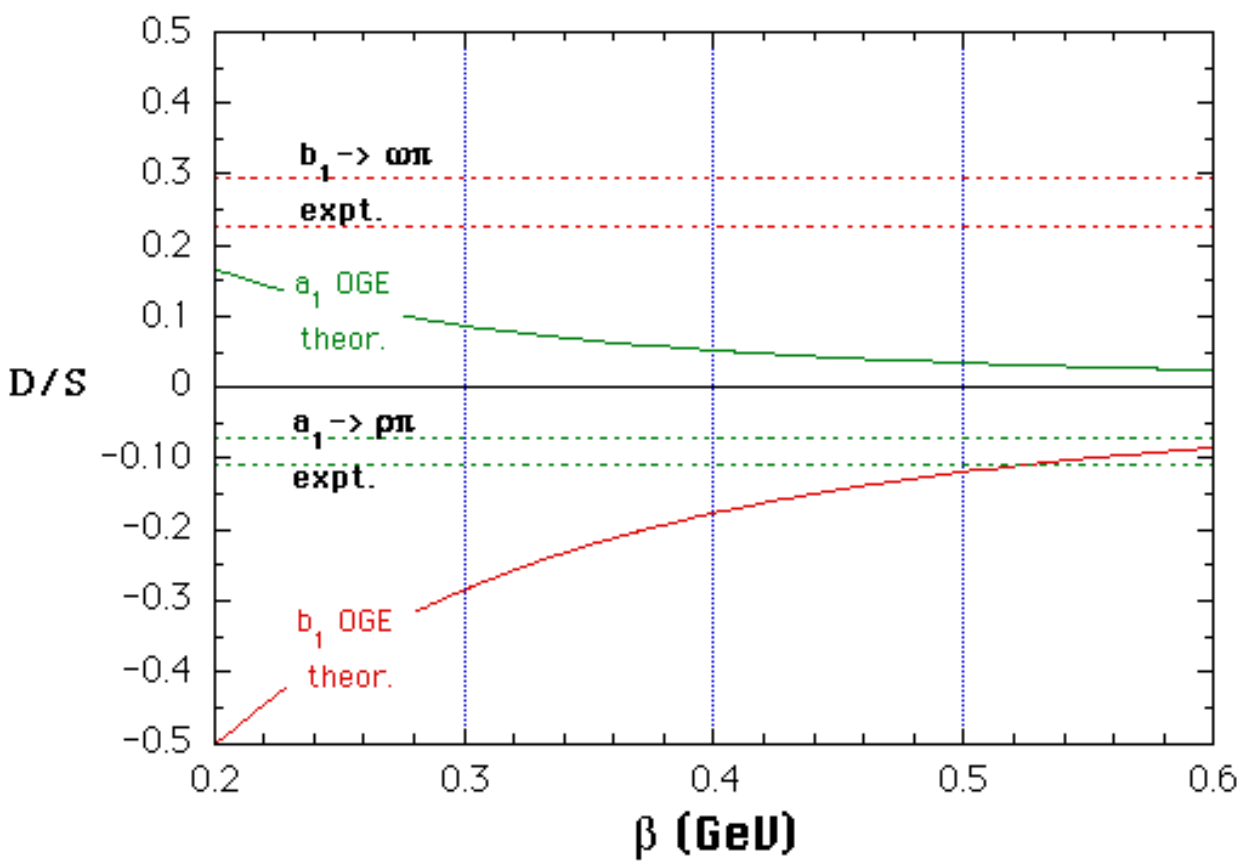

Figure 8. D/S ratios in $b_{1} \rightarrow \omega \pi$ and $a_{1} \rightarrow \rho \pi$ assuming pure OGE decay amplitudes.

In reality both sKs and OGE decay amplitudes are present, and figure 6 shows that the OGE contributions are not negligible here. It is especially interesting to investigate the 
combined effect of sKs and OGE amplitudes, because it may be possible to identify the individual contributions through interference. In the following we set $M\left(a_{1}\right)=M\left(b_{1}\right)=$ $1.23 \mathrm{GeV}$ and $M(\rho)=M(\omega)=0.78 \mathrm{GeV}$ to remove small phase space effects. First in figure 9 we show the D/S ratio in $b_{1} \rightarrow \omega \pi$ as a function of the OGE coupling strength $\alpha_{s}$ for a realistic sKs amplitude $\left(b=0.18 \mathrm{GeV}^{2}\right)$, for several values of the wavefunction scale $\beta$.

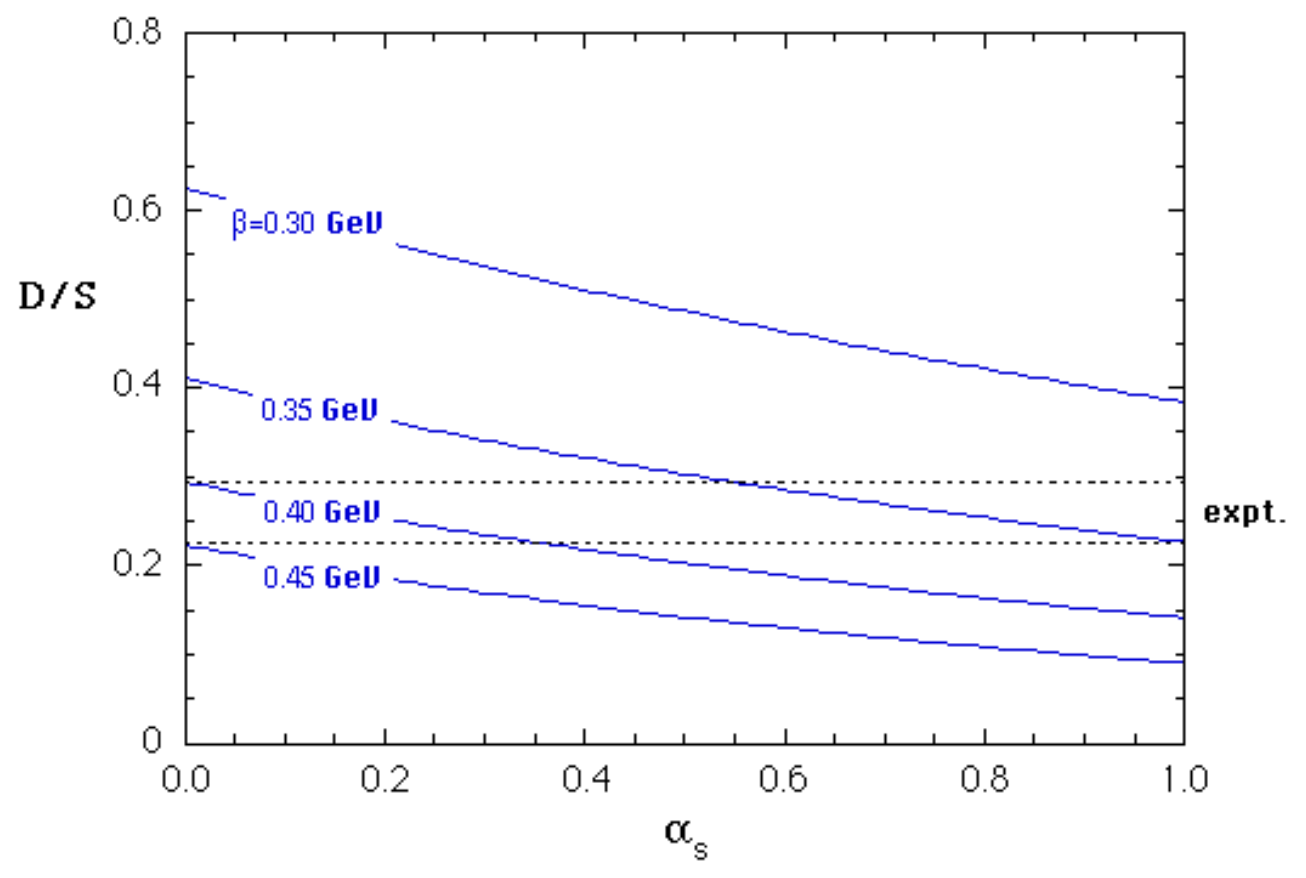

Figure $9 . \mathrm{D} / \mathrm{S}$ ratio in $b_{1} \rightarrow \omega \pi$ with combined sKs and OGE decay amplitudes.

Evidently the effect of OGE is to reduce D/S somewhat, and for the conventional $\alpha_{s}=0.6$ this leads to a more realistic value of the wavefunction scale, $\beta \approx 0.35 \mathrm{GeV}$. Recall that a rather large $\beta \approx 0.45 \mathrm{GeV}$ was required to fit the observed $b_{1}$ and $a_{1} \mathrm{D} / \mathrm{S}$ ratios with the sKs interaction treated in isolation.

A more definitive test of the presence of OGE contributions involves the ratio of $a_{1} / b_{1}$ $\mathrm{D} / \mathrm{S}$ ratios; this is exactly $-1 / 2$ (assuming identical phase space and spatial wavefunctions) 
for any sKs or ${ }^{3} \mathrm{P}_{0}$ decay strength. We show this ratio versus the OGE coupling in figure 10, for the same parameter set as figure 9 .

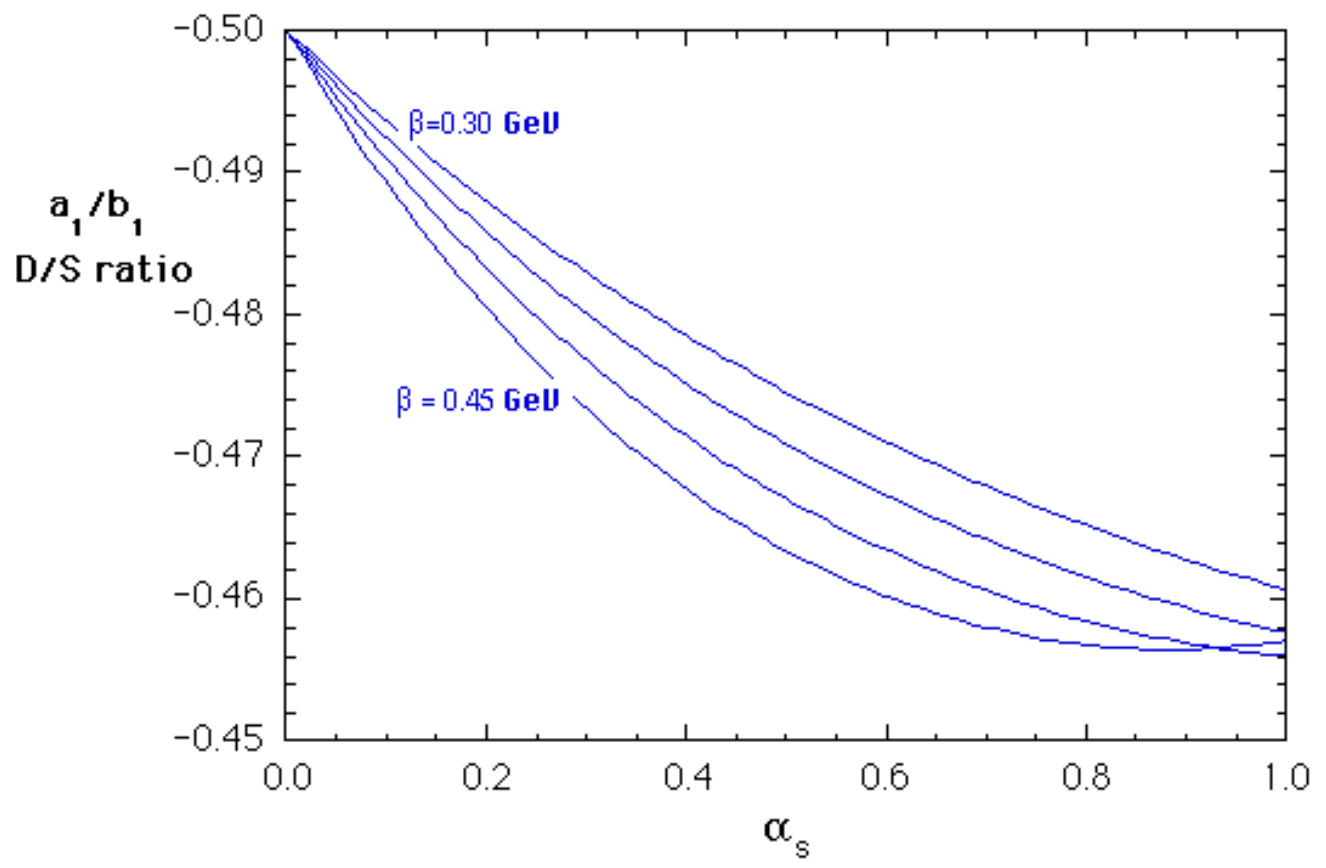

Figure 10. The ratio of $\mathrm{D} / \mathrm{S}$ ratios, $\left(a_{1} \rightarrow \rho \pi\right) /\left(b_{1} \rightarrow \omega \pi\right)$, with combined sKs and OGE decay amplitudes.

For $\alpha_{s}=0.6$ we expect a reduction in the $a_{1} / b_{1} \mathrm{D} / \mathrm{S}$ ratio to $\approx-0.46$ to -0.47 . The current experimental ratio of $-0.35(9)$ suggests a larger departure from the sKs and ${ }^{3} \mathrm{P}_{0}$ prediction of $-1 / 2$, but is consistent with either theoretical result within errors. A more accurate experimental determination of these $\mathrm{D} / \mathrm{S}$ ratios would be interesting as a test of the expected OGE corrections to nonperturbative sKs or ${ }^{3} \mathrm{P}_{0}$ decay amplitudes. 


\section{SUMMARY, CONCLUSIONS AND FUTURE APPLICATIONS.}

In this paper we have discussed the current theoretical understanding of open flavor strong decays and proposed new "microscopic" quark and gluon mechanisms for these decays. We began by reviewing the ${ }^{3} \mathrm{P}_{0}$ decay model and introduced a diagrammatic representation for its decay amplitudes. We then carried out detailed calculations of the decay amplitudes for a representative set of light meson decays, first in the ${ }^{3} \mathrm{P}_{0}$ model and then in microscopic decay models, which assume that the $q \bar{q}$ pair is created by an OGE mechanism or by nonperturbative pair production from the scalar confining potential. These results were derived analytically using well established light quark interactions and SHO wavefunctions.

We find that with conventional quark model parameters the light $q \bar{q}$ open-flavor meson decays are usually dominated by $q \bar{q}$ pair production from the scalar confining potential. We refer to this as the sKs (scalar-kernel-scalar) decay model. This explains the success of the phenomenological ${ }^{3} \mathrm{P}_{0}$ decay model, since the $\bar{\psi} \psi$ scalar current produces pairs in a ${ }^{3} \mathrm{P}_{0}$ state, which is assumed a priori in the ${ }^{3} \mathrm{P}_{0}$ model. The dimensionless pair production strength $\gamma$ is not determined theoretically in the ${ }^{3} \mathrm{P}_{0}$ model, and is treated as a free parameter. In contrast, in our calculations the absolute decay rates are completely determined by the $q \bar{q}$ wavefunction scale $\beta$ and known QCD interaction parameters $\alpha_{s}, b$ (string tension) and $m_{q}$. In the sKs decay model the ${ }^{3} \mathrm{P}_{0}$ strength $\gamma$ corresponds approximately to the dimensionless combination $b / m_{q} \beta$.

Although there are differences in detail between the ${ }^{3} \mathrm{P}_{0}$ and sKs models due to the different overlap integrals, we find that the numerical predictions for relative decay rates and amplitudes in the decays we have considered are remarkably similar. In addition to explaining the success of the ${ }^{3} \mathrm{P}_{0}$ model, our description of decays also accounts naturally for the absence of "hairpin" diagrams; since the microscopic pair production interactions produce $q \bar{q}$ pairs in color-octet states, hairpin diagrams are forbidden without additional interactions. In the usual ${ }^{3} \mathrm{P}_{0}$-like decay models one assumes pair production of a $q \bar{q}$ color 
singlet, and hairpin diagrams are simply ignored without theoretical justification. Other tests of the color state of $q \bar{q}$ pair production in decays would be useful to discriminate between the ${ }^{3} \mathrm{P}_{0}$ model and our color-octet description.

An unsolved problem is that the overall scale of the amplitudes in the full sKs+OGE microscopic decay model is too large by about a factor of two, given conventional quark model parameters. This discrepancy of scale may be due to our neglect of relativistic effects such as the $m_{q} / E$ external line normalizations in (38).

Although we have shown that OGE $q \bar{q}$ pair production is usually dominated by the nonperturbative sKs decay amplitudes, we do find numerically important OGE contributions in some channels, notably ${ }^{3} \mathrm{P}_{0} \rightarrow{ }^{1} \mathrm{~S}_{0}+{ }^{1} \mathrm{~S}_{0}$. In that case OGE pair production actually dominates the nonperturbative sKs amplitude, and insures broad $f_{0}(1300)$ and $\mathrm{K}_{0}^{*}(1430) q \bar{q}$ resonances. OGE pair production also makes characteristic contributions to some observables such as the $\mathrm{D} / \mathrm{S}$ amplitude ratios in $b_{1} \rightarrow \omega \pi$ and $a_{1} \rightarrow \rho \pi$. It may be possible to identify OGE contributions in these and similar multiamplitude decays.

In future work it would be interesting to apply these microscopic decay calculations to charmonium (where the transverse OGE contribution should be much smaller) and to strong decays of light baryons, for which new data is expected from the CEBAF experimental program. The simple exercise of extending these light meson calculations to higher- $\mathrm{L}_{q \bar{q}}$ initial mesons would also be very useful, as it appears likely that large departures from ${ }^{3} \mathrm{P}_{0}$ and sKs model predictions due to OGE contributions could be found among the many decay channels available to these states. 


\section{ACKNOWLEDGMENTS}

We would like to acknowledge useful communications with W.Bardeen, H.Blundell, D.V.Bugg, S.Capstick, F.E.Close, E.Eichten, P.Geiger, S.Godfrey, N.Isgur, A.LeYaouanc, Z.P.Li, D.Lichtenberg, P.R.Page, O.Pene, J.M.Richard, W.Roberts, J.Rosner and N.Törnqvist. We also acknowledge the support of J.Beene of the ORNL Physics Division, and the assistance of M.D.Kovarik with presentation. This research was sponsored in part by the United States Department of Energy under contracts DE-FG02-96ER40944 at North Carolina State University and DE-AC05-840R21400 managed by Lockheed Martin Energy Systems Inc. at Oak Ridge National Laboratory. 
[1] A. LeYaouanc, L.Oliver, O.Pene and J.Raynal, Phys. Rev. D8, 2223 (1973).

[2] ibid., D9, 1415 (1974).

[3] ibid., D11, 1272 (1975).

[4] L.Micu, Nucl. Phys. B10, 521 (1969).

[5] Since the literature is extensive we cite only a few of the original and more recent references. For recent summaries of the ${ }^{3} \mathrm{P}_{0}$ and related decay models see H.G.Blundell and S.Godfrey, Phys. Rev. D53, 3700 (1996) and P.R.Page, "Gluonic Excitations in Mesons", Ph.D. thesis, University of Oxford (1995). For applications to charmonium see A. LeYaouanc, L.Oliver, O.Pene and J.Raynal, Phys. Lett. 71B, 397 (1977); 72B, 57 (1977) and P.R.Page, Nucl. Phys. B446, 189 (1995). For baryon decays see S.Capstick and N.Isgur, Phys. Rev. D34, 2809 (1986) and [11.

[6] P.Geiger and E.S.Swanson, Phys. Rev. D50, 6855 (1994).

[7] R.Kokoski and N.Isgur, Phys. Rev. D35, 907 (1987).

[8] S.Godfrey and N.Isgur, Phys. Rev. D32, 189 (1985).

[9] Several conventions for $\gamma$ are currently in use, which typically differ in the overall normalization of equation (1).

[10] J.W.Alcock, M.J.Burfitt and W.N.Cottingham, Z. Phys. C25, 161 (1984); S. Kumano and V.R. Pandharipande, Phys. Rev. D38, 146 (1988).

[11] S.Capstick and W.Roberts, Phys. Rev. D49, 4570 (1994).

[12] Particle Data Group, Phys. Rev. D50, 1173 (1994).

[13] H.Albrecht et al. (ARGUS Collaboration), Z. Phys. C58, 61 (1993). 
[14] E.Eichten, K.Gottfried, T.Kinoshita, K.D.Lane and T.M.Yan, Phys. Rev. D17, 3090 (1978); Phys. Rev. D23, 203 (1980).

[15] T.Barnes and E.S.Swanson, Phys. Rev. D46, 131 (1992). 


\section{APPENDIX A: DIAGRAMMATIC FORMULATION OF THE ${ }^{3} \mathbf{P}_{0}$ MODEL.}

\section{General results.}

One may simplify calculations of decay amplitudes in the ${ }^{3} \mathrm{P}_{0}$ model by developing a diagrammatic description. This is nonessential for ${ }^{3} \mathrm{P}_{0}$ model calculations of meson decays since there are only two diagrams, but in our generalized decay models there are four diagrams, and it is useful to distinguish their contributions to prove relations between them. We anticipate that the diagrammatic description will also be useful in the more complicated combinatorics of baryon decays.

We begin by noting that the pair production component of the ${ }^{3} \mathrm{P}_{0}$ Hamiltonian (2) can be written in terms of creation operators as

$$
H_{I}=\sum_{s \bar{s}} \int d^{3} k g \frac{m_{q}}{E_{k}}\left[\bar{u}_{\vec{k} s} v_{-\vec{k} \bar{s}}\right] b_{\vec{k} s}^{\dagger} d_{-\vec{k} \bar{s}}^{\dagger} .
$$

We associate both the coupling constant $g$ and the external-line spinor bilinear $\frac{m_{q}}{E_{k}}\left[\bar{u}_{\vec{k} s} v_{-\vec{k}}\right]$ with an effective ${ }^{3} \mathrm{P}_{0} q \bar{q}$ pair production vertex. There is no additional factor of $-i$, unlike conventional field theoretic Feynman rules, because we are determining the matrix element of $H_{I}$ instead of the $T$-matrix.

We assume nonrelativistic $q \bar{q}$ wavefunctions for the initial and final mesons; in our notation a meson state is of the form

$$
|A\rangle=\int d^{3} a \int d^{3} \bar{a} \phi(\vec{a}-\overrightarrow{\vec{a}}) \delta(\vec{A}-\vec{a}-\overrightarrow{\vec{a}})|a \bar{a}\rangle
$$

with implicit spin and flavor wavefunctions that are the usual nonrelativistic quark model

forms. (For details of our conventions for the wavefunctions see reference [15], Appendix A.; note that a factor of $1 /(2 \pi)^{3 / 2}$ was inadvertantly omitted from the normalization of the wavefunction in equation (A15) in that reference.)

One may now evaluate the Hamiltonian matrix element for the decay $\mathrm{A} \rightarrow \mathrm{BC}$ in terms of straightforward overlap integrals. Schematically, we have a matrix element of the type

$$
\left\langle b \bar{b} c \bar{c}\left|b_{\vec{k} s}^{\dagger} d_{-\vec{k} \bar{s}}^{\dagger}\right| a \bar{a}\right\rangle,
$$


and it is useful to distinguish two Feynman diagrams (figure A1) which we refer to as $d_{1}$ if the produced quark goes into meson $\mathrm{B}$ and $d_{2}$ if it goes into $\mathrm{C}$.
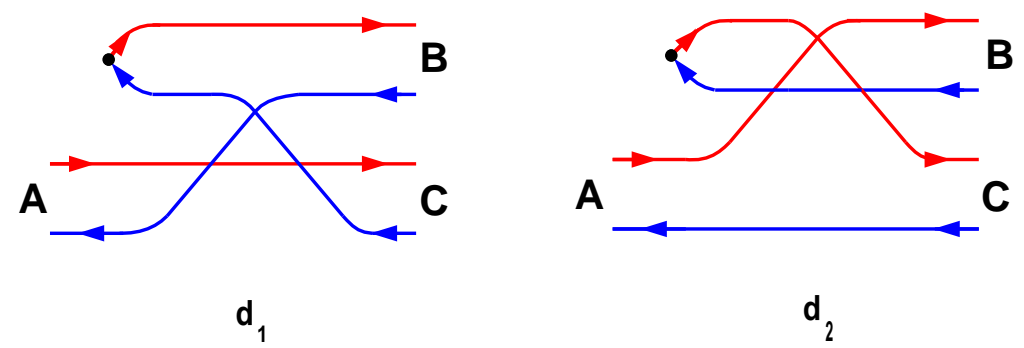

Figure A1. $q \bar{q}$ meson decay diagrams in the ${ }^{3} \mathrm{P}_{0}$ decay model.

These are drawn so the ordered quark and antiquark content of the state can be read from the diagram by drawing a vertical line through it. Thus, in $d_{1}$ the initial state is $|A\rangle=|a \bar{a}\rangle$ but immediately after the pair creation we have $b_{q}^{\dagger} d_{\bar{q}}^{\dagger}|a \bar{a}\rangle=|q \bar{q} a \bar{a}\rangle$. This is then rearranged to $|q \bar{a} a \bar{q}\rangle$, which is projected onto the final state $|B C\rangle=|b \bar{b} c \bar{c}\rangle$. The odd number of crossed lines in each diagram implies a $(-1)$ phase (the "signature" of the matrix element) due to permutation of quark and antiquark operators.

Specializing to diagram $d_{1}$, the associated matrix element is of the form

$$
\left\langle B C\left|H_{I}\right| A\right\rangle_{d_{1}}=I_{\text {signature }} \cdot I_{\text {flavor }} \cdot \mathbf{I}_{\text {spin }+ \text { space }} .
$$

The spatial overlap integral associated with diagram $d_{1}$ (before the spin matrix element is taken, which gives $\left.\mathbf{I}_{\text {spin+space }}\left(d_{1}\right)\right)$ is

$$
\begin{gathered}
\mathbf{I}_{\text {space }}\left(d_{1}\right)=\iiint \iiint d^{3} a d^{3} \bar{a} d^{3} b d^{3} \bar{b} d^{3} c d^{3} \bar{c} \\
\underbrace{\phi(\vec{a}-\overrightarrow{\vec{a}}) \delta(\vec{A}-\vec{a}-\overrightarrow{\vec{a}})}_{\text {meson A }} \underbrace{\phi^{*}(\vec{b}-\overrightarrow{\vec{b}}) \delta(\vec{B}-\vec{b}-\overrightarrow{\vec{b}})}_{\text {meson B }} \underbrace{\phi^{*}(\vec{c}-\overrightarrow{\vec{c}}) \delta(\vec{C}-\vec{c}-\overrightarrow{\vec{c}})}_{\text {meson } \mathrm{C}} \\
\underbrace{\int d^{3} k g \frac{m_{q}}{E_{k}}\left[\bar{u}_{\vec{k} s} v_{-\vec{k} \bar{s}}\right] \delta(\vec{k}-\vec{b}) \delta(-\vec{k}-\overrightarrow{\vec{c}})}_{\text {pair production amplitude }} \underbrace{\delta(\vec{a}-\vec{c}) \delta(\overrightarrow{\vec{a}}-\overrightarrow{\vec{b}})}_{\text {spectator lines }}
\end{gathered}
$$

This result can be read directly from the diagram (see figure A2), using the pair production vertex $(\mathrm{A} 1)$ and the fact that each unscattered "spectator" line gives a factor of $\delta\left(\vec{k}_{i}-\vec{k}_{f}\right)$. 


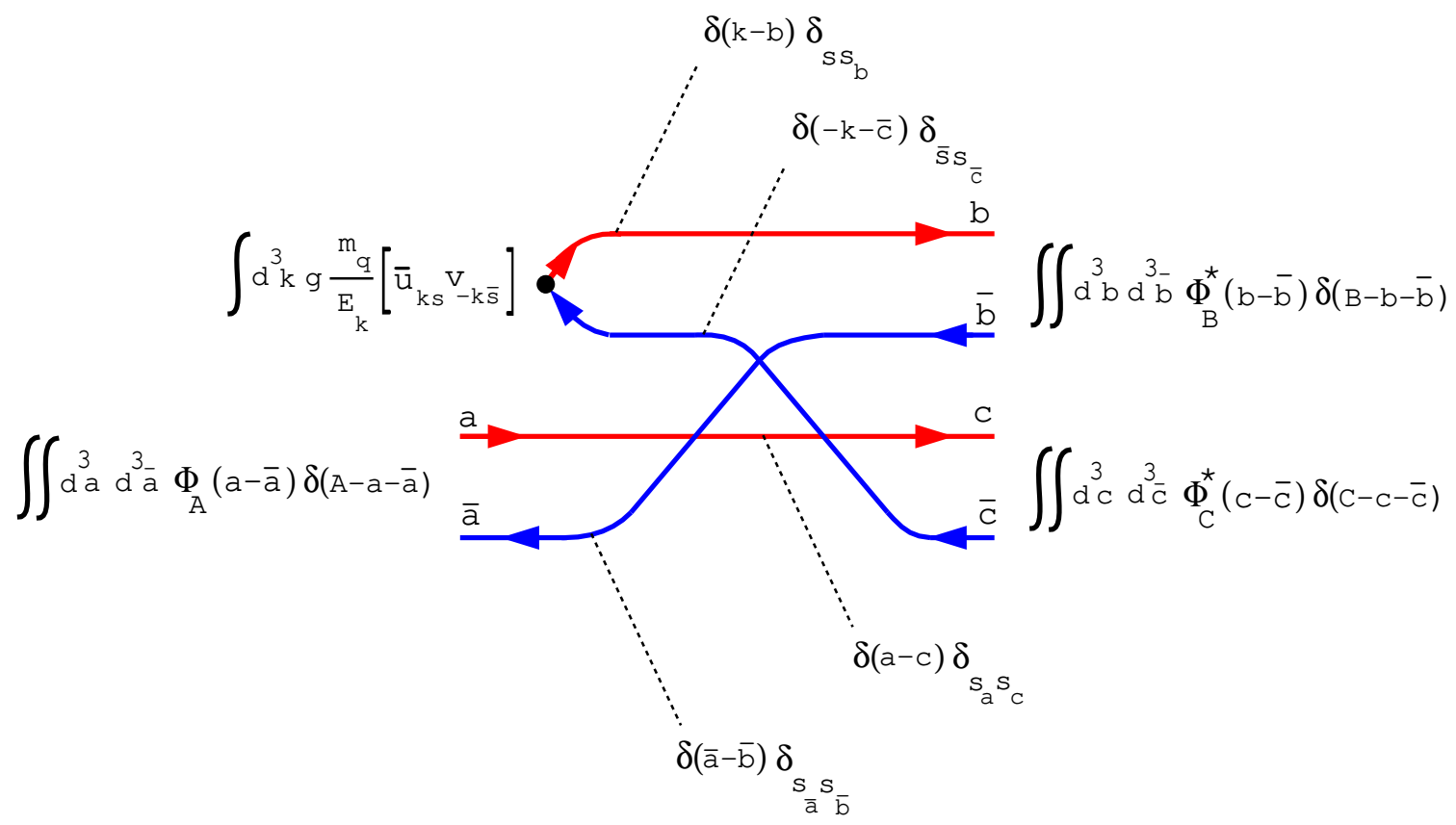

Figure A2. Determination of the spatial overlap integral for diagram $d_{1}$.

Thus the decay amplitude is a 21-dimensional integral involving 21 one-dimensional delta functions. Only 18 of the integrations can be carried out trivially, which leaves a threedimensional overlap integral times a $\delta(\vec{A}-\vec{B}-\vec{C})$ momentum-conserving delta function,

$$
\mathbf{I}_{\text {space }}=I_{\text {space }} \delta(\vec{A}-\vec{B}-\vec{C}),
$$

as in (4). These overlap integrals are explicitly (setting $\vec{A}=0$ and $\vec{B}=-\vec{C}$ )

$$
\begin{aligned}
& I_{\text {space }}\left(d_{1}\right)=\int d^{3} k \phi_{A}(2 \vec{k}-2 \vec{B}) \phi_{B}^{*}(2 \vec{k}-\vec{B}) \phi_{C}^{*}(2 \vec{k}-\vec{B}) \cdot g \frac{m_{q}}{E_{k}}\left[\bar{u}_{\vec{k} s_{b}} v_{-\vec{k} s_{\bar{c}}}\right], \\
& I_{\text {space }}\left(d_{2}\right)=\int d^{3} k \phi_{A}(2 \vec{k}+2 \vec{B}) \phi_{B}^{*}(2 \vec{k}+\vec{B}) \phi_{C}^{*}(2 \vec{k}+\vec{B}) \cdot g \frac{m_{q}}{E_{k}}\left[\bar{u}_{\vec{k} s_{c}} v_{-\vec{k} s_{\bar{b}}}\right] .
\end{aligned}
$$

The spin factor and labels $s_{q}, s_{\bar{q}}$ in these overlaps depend on the reaction being considered, and are determined by the external line labels attached to the diagrams, as illustrated below.

\section{An illustrative ${ }^{3} \mathbf{P}_{0}$ decay: $\rho \rightarrow \pi \pi$.}

Here and in Appendix $\mathrm{C}$ we will use the decay $\rho^{+}(+\hat{z}) \rightarrow \pi^{+} \pi^{o}$ to illustrate our techniques, and simply quote results for other cases in the text. For this decay the flavor states 
are $\left|\rho^{+}\right\rangle=\left|\pi^{+}\right\rangle=-|u \bar{d}\rangle$ and $\left|\pi^{o}\right\rangle=(|u \bar{u}\rangle-|d \bar{d}\rangle) / \sqrt{2}$. Figure A3 shows the evaluation of the flavor factor for diagram $d_{1}$.

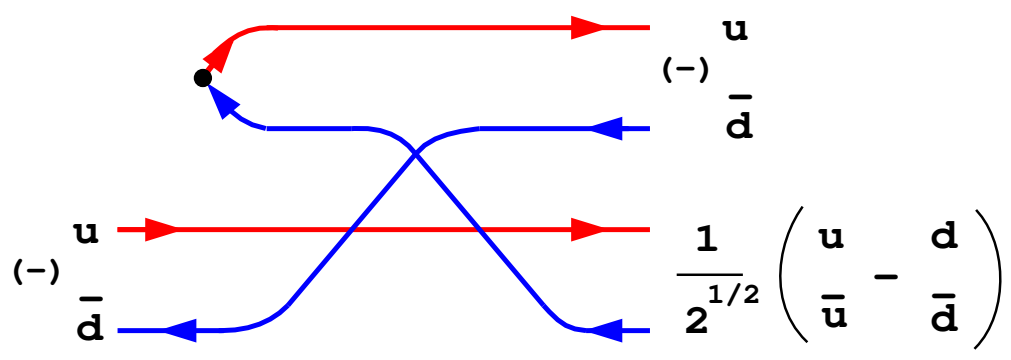

Figure A3. Determination of the flavor factor of $+1 / \sqrt{2}$ for diagram $d_{1}$ in $\rho^{+} \rightarrow \pi^{+} \pi^{o}$.

The flavor factors associated with the other decays we consider can be determined similarly, and are tabulated below. For a given decay, such as $f_{2} \rightarrow \pi \pi$, we give the flavor weight factors for each diagram in a specific charge channel (in this case $f_{2} \rightarrow \pi^{+} \pi^{-}$). The resulting decay rate for $f_{2} \rightarrow \pi^{+} \pi^{-}$must then be multiplied by a multiplicity factor $\mathcal{F}$ to sum over all final flavor states (here $\mathcal{F}=3 / 2$ for $\pi^{+} \pi^{-}$and $\pi^{o} \pi^{o}$ ).

\begin{tabular}{||c|c|c|c|c||}
\hline \multicolumn{5}{||c||}{ Table A1. Flavor Weight Factors. } \\
\hline Generic Decay & Subprocess & $I_{\text {flavor }}\left(d_{1}\right)$ & $I_{\text {flavor }}\left(d_{2}\right)$ & $\mathcal{F}$ \\
\hline$\rho \rightarrow \pi \pi$ & $\rho^{+} \rightarrow \pi^{+} \pi^{o}$ & $+1 / \sqrt{2}$ & $-1 / \sqrt{2}$ & 1 \\
\hline$f \rightarrow \pi \pi$ & $f \rightarrow \pi^{+} \pi^{-}$ & $-1 / \sqrt{2}$ & $-1 / \sqrt{2}$ & $3 / 2$ \\
\hline$a \rightarrow \rho \pi$ & $a^{+} \rightarrow \rho^{+} \pi^{o}$ & $+1 / \sqrt{2}$ & $-1 / \sqrt{2}$ & 2 \\
\hline$b \rightarrow \omega \pi$ & $b^{+} \rightarrow \omega \pi^{+}$ & $+1 / \sqrt{2}$ & $+1 / \sqrt{2}$ & 1 \\
\hline$h \rightarrow \rho \pi$ & $h \rightarrow \rho^{+} \pi^{-}$ & $-1 / \sqrt{2}$ & $-1 / \sqrt{2}$ & 3 \\
\hline $\mathrm{K}^{*} \rightarrow \mathrm{K} \pi$ & $\mathrm{K}^{*+} \rightarrow \mathrm{K}^{+} \pi^{o}$ & $+1 / \sqrt{2}$ & 0 & 3 \\
\hline
\end{tabular}

The "flavorless" decay amplitudes quoted in the text, equations (7-15) and (40-48), correspond to unit flavor factors, $I_{\text {flavor }}\left(d_{1}\right)=+1$ and $I_{\text {flavor }}\left(d_{2}\right)= \pm 1$. (The sign is chosen so the contributions of $d_{1}$ and $d_{2}$ add rather than cancel.) To convert these to physical 
decay amplitudes one should multiply $(7)$ or $(40)$ by $I_{\text {flavor }}\left(d_{1}\right)$ from Table A1, and multiply the resulting total decay rate by $\mathcal{F}$. The $\mathrm{K}^{*}$ is a special case because only one diagram contributes; in this case (7) or (40) should instead be multiplied by $I_{\text {flavor }}\left(d_{1}\right) / 2$.

The spin states are $|\rho(+\hat{z})\rangle=|\uparrow \bar{\uparrow}\rangle$ and $|\pi\rangle=(|\uparrow \bar{\downarrow}\rangle-|\downarrow \uparrow\rangle) / \sqrt{2}$; taking the spin matrix element analogously to figure A3, we find a spin factor of $\left[(-1 / 2) \bar{u}_{\vec{k}, \downarrow} v_{-\vec{k}, \downarrow}\right]$ for this diagram. Combining these results and using the explicit Dirac spinor matrix element in Appendix B, we find

$$
h_{f i}\left(d_{1}\right)=\underbrace{I_{\text {signature }}}_{(-1)} \cdot \underbrace{I_{\text {flavor }}}_{(+1 / \sqrt{2})} \cdot I_{\text {spin }+ \text { space }}\left(d_{1}\right)
$$

where

$$
I_{\text {spin }+ \text { space }}\left(d_{1}\right)=-\frac{1}{2} g \int d^{3} k \phi_{A}(2 \vec{k}-2 \vec{B}) \phi_{B}^{*}(2 \vec{k}-\vec{B}) \phi_{C}^{*}(2 \vec{k}-\vec{B}) \frac{\left(k_{x}+i k_{y}\right)}{E_{k}}
$$

The second diagram $d_{2}$ has an opposite flavor factor, so the full result is

$$
h_{f i}=-\frac{1}{\sqrt{2}}\left(I_{\text {spin }+ \text { space }}\left(d_{1}\right)-I_{\text {spin }+ \text { space }}\left(d_{2}\right)\right) .
$$

Since the overlap integrals satisfy $I\left(d_{2},-\vec{B}\right)=+I\left(d_{1}, \vec{B}\right), h_{f i}$ is odd under $\Omega_{B} \rightarrow-\Omega_{B}$, and can be written as

$$
\begin{gathered}
h_{f i}=-\sqrt{2} I_{\text {spin }+ \text { space }}\left(d_{1}\right) \\
=+\frac{1}{\sqrt{2}} \frac{g}{m_{q}} \int d^{3} k \phi_{A}(2 \vec{k}-2 \vec{B}) \phi_{B}^{*}(2 \vec{k}-\vec{B}) \phi_{C}^{*}(2 \vec{k}-\vec{B})\left(k_{x}+i k_{y}\right) .
\end{gathered}
$$

We have also substituted $m_{q}$ for $E_{k}$ in (A10) to recover the nonrelativistic limit, which gives the ${ }^{3} \mathrm{P}_{0}$ model.

Frequently in the literature these decay amplitudes are evaluated using $\mathrm{SHO} q \bar{q}$ wavefunctions, which leads to closed-form results. For $\mathrm{L}_{q \bar{q}}=0$ mesons our Gaussian momentum space wavefunction $\phi\left(\vec{k}_{q}-\vec{k}_{\bar{q}}\right)$, with $\vec{k}=\vec{k}_{q}=-\vec{k}_{\bar{q}}$, is

$$
\phi(2 \vec{k})=\frac{1}{\pi^{3 / 4} \beta^{3 / 2}} e^{-k^{2} / 2 \beta^{2}}
$$


We note in passing that the $\mathrm{L}_{q \bar{q}}=1$ wavefunction used in this paper is

$$
\phi_{1 m}(2 \vec{k})=\frac{2^{3 / 2}}{3^{1 / 2}} \frac{1}{\pi^{1 / 4} \beta^{3 / 2}} \frac{k}{\beta} e^{-k^{2} / 2 \beta^{2}} Y_{1 m}\left(\Omega_{k}\right) .
$$

On substitution into (A12), $\phi(2 \vec{k})$ gives a $\rho^{+}(+\hat{z}) \rightarrow \pi^{+} \pi^{o}$ decay amplitude of

$$
h_{f i}=-\frac{2^{7 / 2}}{3^{3}} \pi^{-1 / 4} \frac{g}{m_{q}} \frac{P}{\beta^{3 / 2}} e^{-P^{2} / 12 \beta^{2}} Y_{11}\left(\Omega_{B}\right)
$$

and an $\mathcal{M}_{10}^{\rho^{+} \rightarrow \pi^{+} \pi^{o}}$ amplitude of

$$
\mathcal{M}_{10}^{\rho^{+} \rightarrow \pi^{+} \pi^{o}}=-\frac{2^{9 / 2}}{3^{3}} \frac{1}{\pi^{1 / 4} \beta^{1 / 2}} \frac{g}{2 m_{q}} \frac{P}{\beta} e^{-P^{2} / 12 \beta^{2}} Y_{11}\left(\Omega_{B}\right)
$$

Substitution into (6) then gives the total decay rate

$$
\Gamma_{\rho \rightarrow \pi \pi}=\pi^{1 / 2}\left(\frac{2^{10}}{3^{6}}\right)\left(\frac{g}{2 m_{q}}\right)^{2} M_{\rho}(P / \beta)^{3} e^{-P^{2} / 6 \beta^{2}}
$$

which with the identification $\gamma=g / 2 m_{q}$ is the usual ${ }^{3} \mathrm{P}_{0}$ result.

Many features of this decay rate could be anticipated on general grounds. Specifically:

1. The $\left(g / m_{q}\right)^{2}$ dependence follows from the $O(g)$ of the amplitude and the nonrelativistic limit of (2).

2. The factor of $M_{\rho}$ comes from phase space and the relation $E_{B} E_{C} / M_{A}=M_{\rho} / 4$.

3. The $P^{3}$ threshold dependence is expected for a $\mathrm{P}$-wave final state.

4. Dimensional arguments then require a factor of $1 / \beta^{3}$.

5. An exponential in $(P / \beta)^{2}$ is expected given $\mathrm{SHO}$ wavefunctions.

Only the overall numerical coefficient and the factor of $1 / 6$ in the exponential (present in all ${ }^{3} \mathrm{P}_{0} \mathrm{SHO} \mathrm{A} \rightarrow \mathrm{BC} q \bar{q}$ meson decays) require detailed calculation. 


\section{APPENDIX B: OVERLAP INTEGRALS AND SPIN FACTORS.}

In the text we discussed the evaluation of only one of the four decay diagrams present in $\mathrm{A} \rightarrow \mathrm{BC}$ meson decay in JKJ models. Here we give the overlap integrals associated with all four diagrams:

$$
\begin{aligned}
I_{\text {space }}\left(d_{1 q}\right)=+ & \frac{1}{(2 \pi)^{3}} \iint d^{3} a d^{3} c \phi_{A}(2 \vec{a}) \phi_{B}^{*}(2 \vec{a}+\vec{B}) \phi_{C}^{*}(2 \vec{c}+\vec{B}) \\
& {\left[\bar{u}_{b s_{b}} \Gamma v_{\bar{c} s_{\bar{c}}}\right] \mathcal{K}(\vec{a}-\vec{c})\left[\bar{u}_{c s_{c}} \Gamma u_{a s_{a}}\right] }
\end{aligned}
$$

(with implicit momentum constraints specific to this diagram of $\vec{b}=\vec{a}+\vec{B}$ and $\vec{c}=-\vec{B}-\vec{c}$ ),

$$
\begin{gathered}
I_{\text {space }}\left(d_{1 \bar{q}}\right)=-\frac{1}{(2 \pi)^{3}} \iint d^{3} a d^{3} b \phi_{A}(2 \vec{a}) \phi_{B}^{*}(2 \vec{b}-\vec{B}) \phi_{C}^{*}(2 \vec{a}+\vec{B}) \\
{\left[\bar{u}_{b s_{b}} \Gamma v_{\bar{c} \bar{s}_{\bar{c}}}\right] \mathcal{K}(\overrightarrow{\vec{a}}-\overrightarrow{\vec{b}})\left[\bar{v}_{\bar{a} \bar{s}_{\bar{a}}} \Gamma v_{\bar{b}_{\bar{b}}}\right]}
\end{gathered}
$$

(with $\overrightarrow{\vec{c}}=-\vec{B}-\vec{c}, \overrightarrow{\vec{a}}=-\vec{a}$ and $\overrightarrow{\vec{b}}=\vec{B}-\vec{b}$ ),

$$
\begin{aligned}
I_{\text {space }}\left(d_{2 q}\right)=+ & \frac{1}{(2 \pi)^{3}} \iint d^{3} a d^{3} b \phi_{A}(2 \vec{a}) \phi_{B}^{*}(2 \vec{b}-\vec{B}) \phi_{C}^{*}(2 \vec{a}-\vec{B}) \\
& {\left[\bar{u}_{c s_{c}} \Gamma v_{\bar{b}_{\bar{b}}}\right] \mathcal{K}(\vec{a}-\vec{b})\left[\bar{u}_{b s_{b}} \Gamma u_{a s_{a}}\right] }
\end{aligned}
$$

(with $\vec{c}=\vec{a}-\vec{B}$ and $\vec{b}=\vec{B}-\vec{b}$ ),

$$
\begin{aligned}
& I_{\text {space }}\left(d_{2 \bar{q}}\right)=- \frac{1}{(2 \pi)^{3}} \iint d^{3} a d^{3} c \phi_{A}(2 \vec{a}) \phi_{B}^{*}(2 \vec{a}-\vec{B}) \phi_{C}^{*}(2 \vec{c}+\vec{B}) \\
& {\left[\bar{u}_{c s_{c}} \Gamma v_{\bar{b} s_{\bar{b}}}\right] \mathcal{K}(\overrightarrow{\vec{a}}-\overrightarrow{\vec{c}})\left[\bar{v}_{\bar{a} s_{\bar{a}}} \Gamma v_{\bar{c} s_{\bar{c}}}\right] }
\end{aligned}
$$

(with $\vec{a}=-\vec{a}, \vec{b}=\vec{B}-\vec{a}$ and $\vec{c}=-\vec{B}-\vec{c}$ ).

In our evaluation of decay matrix elements in JKJ models we also require spin matrix elements, which involve the nonrelativistic $O(p / m)$ matrix elements of Dirac bilinears with $\Gamma=\gamma^{0}, \vec{\gamma}$ and I and Pauli spin matrix elements for various spin states. These are 


$$
\begin{aligned}
& \lim _{v / c \rightarrow 0}\left[\bar{u}_{q^{\prime} s^{\prime}} \Gamma u_{q s}\right]= \begin{cases}\delta_{s s^{\prime}} & \Gamma=\gamma^{0} \\
\frac{1}{2 m_{q}}\left[\left(\vec{q}+\vec{q}^{\prime}\right) \delta_{s s^{\prime}}-i\left\langle s^{\prime}|\vec{\sigma}| s\right\rangle \times\left(\vec{q}^{\prime}-\vec{q}\right)\right] & \Gamma=\vec{\gamma} \\
\delta_{s s^{\prime}} & \Gamma=\mathrm{I}\end{cases} \\
& \lim _{v / c \rightarrow 0}\left[\bar{u}_{q s} \Gamma v_{\bar{q} \bar{s}}\right]= \begin{cases}\frac{1}{2 m_{q}}\langle s \bar{s}|\vec{\sigma}| 0\rangle \cdot(\overrightarrow{\vec{q}}+\vec{q}) & \Gamma=\gamma^{0} \\
\langle s \bar{s}|\vec{\sigma}| 0\rangle & \Gamma=\vec{\gamma} \\
\frac{1}{2 m_{q}}\langle s \bar{s}|\vec{\sigma}| 0\rangle \cdot(\overrightarrow{\vec{q}}-\vec{q}) & \Gamma=\mathrm{I}\end{cases} \\
& \lim _{v / c \rightarrow 0}\left[\bar{v}_{\bar{q} \bar{s}} \Gamma v_{\bar{q}^{\prime} \bar{s}^{\prime}}\right]= \begin{cases}\delta_{\bar{s} \bar{s}^{\prime}} & \Gamma=\gamma^{0} \\
\frac{1}{2 m_{q}}\left[\left(\overrightarrow{\vec{q}}+\overrightarrow{\bar{q}}^{\prime}\right) \delta_{\bar{s}^{\prime}}-i\left\langle\bar{s}^{\prime}|\vec{\sigma}| \bar{s}\right\rangle \times\left(\overrightarrow{\vec{q}}^{\prime}-\overrightarrow{\vec{q}}\right)\right] & \Gamma=\vec{\gamma} \\
-\delta_{\bar{s} \bar{s}^{\prime}} & \Gamma=\mathrm{I}\end{cases}
\end{aligned}
$$

The explicit scattering and pair-production matrix elements of Pauli spinors in terms of the spherical basis vectors $\hat{e}_{ \pm}=\mp(\hat{x}+i \hat{y}) / \sqrt{2}$ and $\hat{e}_{0}=\hat{z}$, are

$$
\begin{gathered}
\langle\uparrow|\vec{\sigma}| \uparrow\rangle=-\langle\downarrow|\vec{\sigma}| \downarrow\rangle=\hat{e}_{0} \\
\langle\uparrow|\vec{\sigma}| \downarrow\rangle=\sqrt{2} \hat{e}_{-} \\
\langle\downarrow|\vec{\sigma}| \uparrow\rangle=-\sqrt{2} \hat{e}_{+} \\
\langle\uparrow|\vec{\sigma}| \uparrow\rangle=-\langle\bar{\downarrow}|\vec{\sigma}| \bar{\downarrow}\rangle=-\hat{e}_{0} \\
\langle\uparrow|\vec{\sigma}| \bar{\downarrow}\rangle=\sqrt{2} \hat{e}_{+} \\
\langle\bar{\downarrow}|\vec{\sigma}| \uparrow\rangle=-\sqrt{2} \hat{e}_{-} \\
\langle\uparrow \bar{\tau}|\vec{\sigma}| 0\rangle=\sqrt{2} \hat{e}_{-} \\
\langle\uparrow \bar{\downarrow}|\vec{\sigma}| 0\rangle=\langle\downarrow|\vec{\sigma}| 0\rangle=-\hat{e}_{0} \\
\langle\downarrow \bar{\downarrow}|\vec{\sigma}| 0\rangle=\sqrt{2} \hat{e}_{+}
\end{gathered}
$$




\section{APPENDIX C: $\rho \rightarrow \pi \pi$ IN JKJ DECAY MODELS}

We will illustrate a JKJ decay calculation in detail using the reaction $\rho^{+}(+\hat{z}) \rightarrow \pi^{+} \pi^{o}$, as in our discussion of the ${ }^{3} \mathrm{P}_{0}$ model in Appendix A. We will evaluate the $\rho \rightarrow \pi \pi$ decay rate using the $\mathrm{j}^{0} \mathrm{Kj}{ }^{0}$ interaction in (27-29), and simply quote results in the text for the other cases. For each type of JKJ interaction we find that the four diagrams give equal contributions, so we discuss only diagram $d_{1 q}$, shown below, in detail.

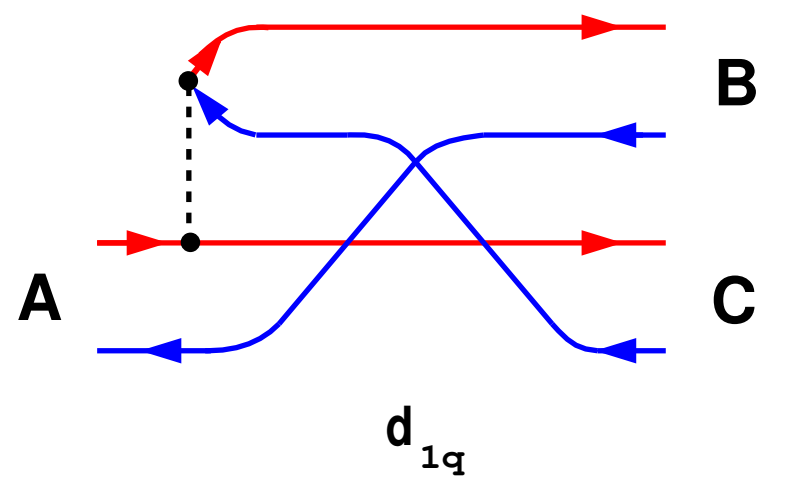

Figure C1. Decay diagram $d_{1 q}$.

For all the JKJ interactions we consider this diagram has a signature of $(-1)$, a color factor of $+2^{2} / 3^{3 / 2}$, and a flavor factor (for $\rho^{+} \rightarrow \pi^{+} \pi^{o}$ ) of $+1 / 2^{1 / 2}$. Differences in the relative importance of the decay mechanisms arise in the spin-space overlap integrals and the $\Gamma$ spinor matrix elements.

In the text we derived the spatial overlap integral for diagram $d_{1 q}$ in the nonrelativistic limit, equation (39);

$$
\begin{aligned}
I_{\text {space }}\left(d_{1 q}\right)= & \frac{1}{(2 \pi)^{3}} \iint d^{3} a d^{3} c \phi_{A}(2 \vec{a}) \phi_{B}^{*}(2 \vec{a}+\vec{B}) \phi_{C}^{*}(2 \vec{c}+\vec{B}) \\
& {\left[\bar{u}_{b s_{b}} \Gamma v_{\bar{c} s_{\bar{c}}}\right] \mathcal{K}(|\vec{a}-\vec{c}|)\left[\bar{u}_{c s_{c}} \Gamma u_{a s_{a}}\right] . }
\end{aligned}
$$

To evaluate this for $\rho^{+}(+\hat{z}) \rightarrow \pi^{+} \pi^{o}$, note that for the color Coulomb interaction $\Gamma=\gamma^{0}$ the spinor bilinears give 


$$
\left[\bar{u}_{b s_{b}} \gamma^{0} v_{\bar{c} s_{\bar{c}}}\right]\left[\bar{u}_{c s_{c}} \gamma^{0} u_{a s_{a}}\right]=\frac{1}{2 m_{q}}\left\langle s_{b} s_{\bar{c}}|\vec{\sigma}| 0\right\rangle \cdot(\vec{b}+\overrightarrow{\bar{c}}) \delta_{s_{c} s_{a}}
$$

Attaching the spin wavefunctions to the diagram $d_{1 q}$ gives an overall factor of $(-1 / 2)$ and sets $s_{b}=\downarrow$ and $s_{\bar{c}}=\bar{\downarrow}$, so the spin matrix element is $\langle\vec{\sigma}\rangle_{q \bar{q}}=(-1 / 2)\langle\downarrow \bar{\downarrow}|\vec{\sigma}| 0\rangle=-\hat{e}_{+} / \sqrt{2}$. With the substitutions $\vec{Q}=(\vec{a}-\vec{c})=(\vec{b}+\overrightarrow{\vec{c}})$ and $\vec{\Sigma}=(\vec{a}+\vec{c}) / 2, h_{f i}$ becomes

$$
\begin{gathered}
h_{f i}\left(d_{1 q}\right)=\underbrace{(-1)}_{\text {signature }} \underbrace{\left(+\frac{2^{2}}{3^{3 / 2}}\right)}_{\text {color }} \underbrace{\left(+\frac{1}{2^{1 / 2}}\right)}_{\text {flavor }} \\
\cdot \iint \frac{d^{3} Q d^{3} \Sigma}{(2 \pi)^{3}} \frac{\langle\vec{\sigma}\rangle_{q \bar{q}} \cdot \vec{Q}}{2 m_{q}} \mathcal{K}(Q) \phi_{\rho}(2 \vec{\Sigma}+\vec{Q}) \phi_{\pi^{+}}^{*}(2 \vec{\Sigma}+\vec{Q}+\vec{B}) \phi_{\pi^{o}}^{*}(2 \vec{\Sigma}-\vec{Q}+\vec{B})
\end{gathered}
$$

Without additional information about the spatial wavefunctions this is the final result. To proceed we assume standard quark model Gaussian wavefunctions (A13) for the mesons. The $d^{3} \Sigma$ integral can then be carried out, which gives

$$
\begin{aligned}
& h_{f i}\left(d_{1 q}\right)=-2^{-1} 3^{-3} \pi^{-15 / 4} m_{q}^{-1} \beta^{-3 / 2} e^{-x^{2} / 16} \\
& \cdot \underbrace{\int d^{3} Q\langle\vec{\sigma}\rangle_{q \bar{q}} \cdot \vec{Q} \mathcal{K}(Q) \exp \left\{-\frac{1}{3 \beta^{2}}\left(\vec{Q}-\frac{1}{4} \vec{B}\right)^{2}\right\}}_{I}
\end{aligned}
$$

where we have again abbreviated $P / \beta=x$.

We may evaluate this intermediate integral $I$ by returning to coordinate space using (37) and integrating over $d^{3} Q$, which gives

$$
I=3^{3 / 2} \pi^{3 / 2} \beta^{3} \int d^{3} r\langle\vec{\sigma}\rangle_{q \bar{q}} \cdot\left(\frac{1}{4} \vec{B}+\frac{3 i}{2} \beta^{2} \vec{r}\right) K(r) e^{\frac{i}{4} \vec{B} \cdot \vec{r}} e^{-3 \beta^{2} r^{2} / 4}
$$

For $\langle\vec{\sigma}\rangle_{q \bar{q}}$ in this reaction we have

$$
\langle\vec{\sigma}\rangle_{q \bar{q}} \cdot \vec{r}=-\sqrt{\frac{2 \pi}{3}} r Y_{11}(\Omega)
$$

and the angular integrals can then be carried out using a spherical harmonic expansion of the plane wave in (C4). This gives

$$
I=-2^{5 / 2} 3 \pi^{3} \beta^{3} Y_{11}\left(\Omega_{B}\right) \int_{0}^{\infty} r^{2} d r K(r) e^{-3 \beta^{2} r^{2} / 4}\left[\frac{1}{4} P j_{0}(\operatorname{Pr} / 4)-\frac{3}{2} \beta^{2} r j_{1}(\operatorname{Pr} / 4)\right] .
$$


Since the kernel is simply $K(r)=\alpha_{s} / r$ in this case, the radial integrals can be evaluated in terms of confluent hypergeometric functions using the general formula

$$
\int_{0}^{\infty} d r r^{n} j_{\ell}(a r) e^{-b r^{2}}=\sqrt{\pi} \frac{a^{\ell}}{b^{\phi}} \frac{\Gamma(\phi)}{2^{\ell+2} \Gamma(\ell+3 / 2)}{ }_{1} \mathrm{~F}_{1}\left(\frac{\ell-n}{2}+1 ; \ell+\frac{3}{2} ; \frac{a^{2}}{4 b}\right) e^{-a^{2} / 4 b}
$$

where $\phi=(n+\ell+1) / 2$. This result suffices for all the overlap integrals we encounter in JKJ decay models with SHO wavefunctions and power-law kernels.

Applied to the Coulomb case, our result for the integral $I$ is

$$
I=-2^{3 / 2} \pi^{3} \alpha_{s} \beta^{2} x\left[{ }_{1} \mathrm{~F}_{1}\left(\frac{1}{2} ; \frac{3}{2} ; \xi\right)-\frac{2}{3}{ }_{1} \mathrm{~F}_{1}\left(\frac{1}{2} ; \frac{5}{2} ; \xi\right)\right] e^{-x^{2} / 48} Y_{11}\left(\Omega_{B}\right)
$$

where $\xi=x^{2} / 48$. Using this integral we obtain our final result for diagram $d_{1 q}$,

$$
h_{f i}\left(d_{1 q}\right)=\frac{2^{1 / 2}}{3^{3}} \pi^{-3 / 4} \frac{\alpha_{s}}{m_{q}} \beta^{1 / 2} x\left[{ }_{1} \mathrm{~F}_{1}\left(\frac{1}{2} ; \frac{3}{2} ; \xi\right)-\frac{2}{3}{ }_{1} \mathrm{~F}_{1}\left(\frac{1}{2} ; \frac{5}{2} ; \xi\right)\right] e^{-x^{2} / 12} Y_{11}\left(\Omega_{B}\right) .
$$

On multiplying by 4 for the four equivalent diagrams, we obtain the full result for $h_{f i}$ for $\rho^{+}(+\hat{z}) \rightarrow \pi^{+} \pi^{o}$. We can abstract the $\mathrm{j}^{0} \mathrm{Kj}^{0}$ decay amplitude $\mathcal{M}_{L S}$ in (6) from this; removing the $\rho^{+} \rightarrow \pi^{+} \pi^{o}$ flavor factor of $1 / \sqrt{2}$, we have

$$
\mathcal{M}_{10}^{\left({ }^{3} \mathrm{~S}_{1} \rightarrow{ }^{1} \mathrm{~S}_{0}+{ }^{1} \mathrm{~S}_{0}\right)}=+\frac{2^{3}}{3^{3}} \pi^{-3 / 4} \frac{\alpha_{s}}{m_{q}} \beta^{1 / 2} x\left[{ }_{1} \mathrm{~F}_{1}\left(\frac{1}{2} ; \frac{3}{2} ; \xi\right)-\frac{2}{3}{ }_{1} \mathrm{~F}_{1}\left(\frac{1}{2} ; \frac{5}{2} ; \xi\right)\right] e^{-x^{2} / 12}
$$

which is equivalent to the result quoted in (41). Using (6), we find that the total $\rho \rightarrow \pi \pi$ decay rate due to color Coulomb OGE (treated in isolation) is

$$
\Gamma_{\rho \rightarrow \pi \pi}^{\mathrm{j}^{0} \mathrm{Kj}{ }^{0}}=\pi^{-1 / 2}\left(\frac{2^{6}}{3^{6}}\right) \alpha_{s}^{2}\left(\frac{\beta}{m_{q}}\right)^{2} \frac{E_{\pi}^{2}}{M_{\rho}} x^{3}\left[{ }_{1} \mathrm{~F}_{1}\left(\frac{1}{2} ; \frac{3}{2} ; \xi\right)-\frac{2}{3}{ }_{1} \mathrm{~F}_{1}\left(\frac{1}{2} ; \frac{5}{2} ; \xi\right)\right]^{2} e^{-x^{2} / 6} .
$$

Alternatively, for the specific case of a Coulomb kernel we can evaluate the momentum space integral (C3) directly. The required integrals and others needed to evaluate decay amplitudes for P-wave quarkonia are given below.

$$
\begin{aligned}
& \int d^{3} Q\left(1 / \vec{Q}^{2}\right) e^{-a\left(\vec{Q}-\vec{Q}_{0}\right)^{2}}=\frac{\pi^{3 / 2}}{a^{1 / 2}}\left\{2{ }_{1} \mathrm{~F}_{1}\left(\frac{1}{2}, \frac{3}{2}, a \vec{Q}_{0}^{2}\right)\right\} e^{-a \vec{Q}_{0}^{2}}, \\
& \int d^{3} Q\left(Q_{i} / \vec{Q}^{2}\right) e^{-a\left(\vec{Q}-\vec{Q}_{0}\right)^{2}}=\frac{\pi^{3 / 2}}{a^{1 / 2}}\left\{\frac{2}{3} Q_{0 i}{ }_{1} F_{1}\left(\frac{3}{2}, \frac{5}{2}, a \vec{Q}_{0}^{2}\right)\right\} e^{-a \vec{Q}_{0}^{2}},
\end{aligned}
$$




$$
\begin{aligned}
& \int d^{3} Q\left(Q_{i} Q_{j} / \vec{Q}^{2}\right) e^{-a\left(\vec{Q}-\vec{Q}_{0}\right)^{2}}= \\
& \frac{\pi^{3 / 2}}{a^{1 / 2}}\left\{\frac{1}{3 a} \delta_{i j} \mathrm{~F}_{1}\left(\frac{3}{2}, \frac{5}{2}, a \vec{Q}_{0}^{2}\right)+\frac{2}{5} Q_{0 i} Q_{0 j} \mathrm{~F}_{1}\left(\frac{5}{2}, \frac{7}{2}, a \vec{Q}_{0}^{2}\right)\right\} e^{-a \vec{Q}_{0}^{2}}, \\
& \int d^{3} Q\left(Q_{i} Q_{j} / \vec{Q}^{4}\right) e^{-a\left(\vec{Q}-\vec{Q}_{0}\right)^{2}}= \\
& \frac{\pi^{3 / 2}}{a^{1 / 2}}\left\{\frac{2}{3} \delta_{i j} \mathrm{~F}_{1}\left(\frac{1}{2}, \frac{5}{2}, a \vec{Q}_{0}^{2}\right)+\frac{4}{15} a Q_{0 i} Q_{0 j} \mathrm{~F}_{1}\left(\frac{3}{2}, \frac{7}{2}, a \vec{Q}_{0}^{2}\right)\right\} e^{-a \vec{Q}_{0}^{2}}, \\
& \int d^{3} Q\left(Q_{i} Q_{j} Q_{k} / \vec{Q}^{4}\right) e^{-a\left(\vec{Q}-\vec{Q}_{0}\right)^{2}}= \\
& \frac{\pi^{3 / 2}}{a^{1 / 2}}\left\{\frac{2}{15}\left(\delta_{i j} Q_{0 k}+\delta_{j k} Q_{0 i}+\delta_{k i} Q_{0 j}\right){ }_{1} \mathrm{~F}_{1}\left(\frac{3}{2}, \frac{7}{2}, a \vec{Q}_{0}^{2}\right)\right. \\
&\left.\quad+\frac{4}{35} a Q_{0 i} Q_{0 j} Q_{0 k} \mathrm{~F}_{1}\left(\frac{5}{2}, \frac{9}{2}, a \vec{Q}_{0}^{2}\right)\right\} e^{-a \vec{Q}_{0}^{2}} .
\end{aligned}
$$

This approach applied to $\rho \rightarrow \pi \pi$ leads to the form

$$
\Gamma_{\rho \rightarrow \pi \pi}^{\mathrm{j}^{0} \mathrm{Kj}}{ }^{0}=\pi^{-1 / 2}\left(\frac{2^{6}}{3^{8}}\right) \alpha_{s}^{2}\left(\frac{\beta}{m_{q}}\right)^{2} \frac{E_{\pi}^{2}}{M_{\rho}} x^{3}{ }_{1} \mathrm{~F}_{1}\left(\frac{3}{2} ; \frac{5}{2} ; \xi\right)^{2} e^{-x^{2} / 6}
$$

which is equivalent to (C12).

Relations between confluent hypergeometric functions with different indices allow these results to be written in various forms. We typically express our final results for decay amplitudes as linear combinations of confluent hypergeometric functions with constant coefficients and a common first index $a$, times a centrifical factor of $x^{L_{B C}}$. These rearrangements are straightforward using the recurrence relations

$$
{ }_{1} \mathrm{~F}_{1}(a ; c ; x)=\frac{(c-1)}{(a-1)}{ }_{1} \mathrm{~F}_{1}(a-1 ; c-1 ; x)+\frac{(a-c)}{(a-1)}{ }_{1} \mathrm{~F}_{1}(a-1 ; c ; x)
$$

and

$$
x_{1} \mathrm{~F}_{1}(a ; c ; x)=\frac{(c-1)(c-2)}{(a-1)}\left\{{ }_{1} \mathrm{~F}_{1}(a-1 ; c-2 ; x)-{ }_{1} \mathrm{~F}_{1}(a-1 ; c-1 ; x)\right\} .
$$

The decay rates due to transverse OGE and the confining interaction may be derived using the same techniques, with the minor complication that transverse OGE also has initialline spin-flip contributions. These results are quoted in Section II.D. 